\title{
Local Existence for Solutions of Fully Non-linear Wave Equations
}

\author{
Peter Lesky Jr \\ Mathematisches Institut A, Universität Stuttgart, Pfaffenwaldring 57, W-7000, Stuttgart 80, \\ Germany
}

Communicated by $\mathbf{R}$. Leis

Let $\Omega$ be a domain in $\mathbb{R}^{n}$ and let $m \in \mathbb{N}$ be given. We study the initial-boundary value problem for the equation

$$
F\left(t, x, \bar{D}_{x}^{2 m} u(t, x), \bar{D}_{x}^{m} \partial_{t} u(t, x), \partial_{t}^{2} u(t, x)\right)=f(t, x)
$$

with a homogeneous Dirichlet boundary condition; here $u$ is a scalar function, $\bar{D}_{x}^{m} u:=\left(\partial_{x}^{\alpha} u\right)_{|x| \leqslant m}$ and certain restrictions are made on $F$ guaranteeing that energy estimates are possible. We prove the existence of a value of $T>0$ such that a unique classical solution $u$ exists on $[0, T] \times \Omega$. Furthermore, we show that $T \rightarrow \infty$ if the data tend to zero.

\section{Introduction}

Let $\Omega$ be a domain in $\mathbb{R}^{n}$ and let $m \in \mathbb{N}$ be given. For every sufficiently smooth function $u$ defined on $[0, T] \times \Omega$ we set

$$
\begin{aligned}
\bar{D}_{x}^{m} u(t, x) & :=\left(\partial_{x}^{\alpha} u(t, x)=\frac{\partial^{|\alpha|} u(t, x)}{\partial x_{1}^{\alpha_{1}} \cdots \partial x_{n}^{\alpha_{n}}}:|\alpha|=\alpha_{1}+\cdots+\alpha_{n} \leqslant m\right), \\
\mathscr{D} u(t, x) & :=\left(\bar{D}_{x}^{2 m} u(t, x), \bar{D}_{x}^{m} \partial_{t} u(t, x), \partial_{t}^{2} u(t, x)\right) .
\end{aligned}
$$

In this paper we study for a scalar function $u$ the mixed problem

$$
\begin{array}{ll}
F(t, x, \mathscr{D} u(t, x))=f(t, x) & \text { in }[0, T] \times \Omega, \\
\bar{D}_{x}^{m-1} u(t, x)=0 & \text { on }[0, T] \times \partial \Omega, \\
u(0, x)=u^{0}(x), \quad \partial_{t} u(0, x)=u^{1}(x) & \text { in } \Omega
\end{array}
$$

under certain restrictions on $F$ guaranteeing that (1.1) is a non-linear wave equation. For example,

$$
F(t, x, \mathscr{D} u(t, x))=\partial_{t}^{2} u(t, x)-\sum_{|\alpha| \leqslant 2} a_{\alpha}\left(t, x, \bar{D}_{x}^{2} u(t, x)\right) \partial_{x}^{\alpha} u(t, x),
$$

where the coefficient $a_{\alpha}$ have to obey a special ellipticity condition (compare (2.16)). 
The restrictions made on $F$ can be roughly described as follows. Differentiating (1.1) with respect to $t$ and dividing the result by $\partial F / \partial\left(\partial_{1}^{2} u\right)$ we obtain

$$
\partial_{t}^{3} u(t, x)+\mathscr{A}_{u}(t) \partial_{t} u(t, x)+\mathscr{B}_{u}(t) \partial_{t}^{2} u(t, x)=g_{u}(t, x)
$$

in $[0, T] \times \Omega ;$ here $\mathscr{A}_{u}(t)$ and $\mathscr{B}_{u}(t)$ denote families of spatial operators of order $2 m$ and $m$, respectively, having coefficients depending on $(t, x, \mathscr{D} u(t, x))$, and $g_{u}$ is suitably defined (compare (2.7)-(2.11)). We suppose that $\mathscr{A}_{u}(t)$ is uniformly strongly elliptic for every $t \in[0, T]$. Furthermore, we assume that there exists a (not necessarily positive) constant $c \in \mathbb{R}$ such that

$$
\operatorname{Re}\left\langle\mathscr{B}_{u}(t) \varphi, \varphi\right\rangle \geqslant c\|\varphi\|^{2} \text { for } \varphi \in \stackrel{\circ}{H}^{m}(\Omega), \quad t \in[0, T],
$$

where $\langle.,$.$\rangle and \|$.$\| denote the inner product and the norm in L_{2}(\Omega)$, and $\stackrel{\circ}{H}^{m}(\Omega)$ denotes the closure of $C_{0}^{\infty}(\Omega)$ in the $m$ th Sobolev space $H^{m}(\Omega)$. We mention that (1.6) holds in the case $m=1$ if only $F$ is supposed to be real valued. For details compare section 2 and especially Assumption 2.1.

We prove the existence of a $T>0$ such that (1.1)-(1.3) has a unique solution

$$
u \in \mathscr{C}_{T}^{k}:=\bigcap_{j=\mathbf{0}}^{k} C^{j}\left([0, T], H^{(k-j) m}(\Omega)\right)
$$

with $k \geqslant[n / 2 m]+6([r]:=\max \{k \in \mathbb{N}: k \leqslant r\})$. Then $u$ is a classical solution (compare Lemma 5.1). For functions $u \in \mathscr{C}_{T}^{*}$ we understand (1.2) in the sense that

$$
u(t)=u(t, .) \in \stackrel{\circ}{H}^{m}(\Omega) \text { for } t \in[0, T] .
$$

We require that the solution $u$ of $(1.1)-(1.3)$ satisfies

$$
\sup _{[0, T] \times \bar{\Omega}} \mathscr{D} u(t, x) \mid \leqslant M
$$

for a given $M>0$, since the properties of $\mathscr{A}_{u}$ and $\mathscr{B}_{u}$ mentioned above will only be supposed if $u$ satisfies (1.9). In order to guarantee (1.9) we have to assume that the data (and $F$ ) are suitable.

Definition 1.1. Let $u^{0} \in C^{2 m}(\bar{\Omega}), u^{1} \in C^{m}(\bar{\Omega})$ and $f \in C([0, T] \times \bar{\Omega})$. We say that $\left(u^{0}, u^{1}, f\right) \in \mathscr{I}$ if and only if there exists a $u^{2} \in C(\bar{\Omega})$ such that

$$
\begin{aligned}
& F\left(0, x, \bar{D}_{x}^{2 m} u^{0}(x), \bar{D}_{x}^{m} u^{1}(x), u^{2}(x)\right)=f(0, x) \text { in } \Omega, \\
& \sup _{x \in \bar{\Omega}}\left(\left|\bar{D}_{x}^{2 m} u^{0}(x)\right|+\left|\bar{D}_{x}^{m} u^{1}(x)\right|+\left|u^{2}(x)\right|\right) \leqslant \frac{1}{2} M .
\end{aligned}
$$

Now we can formulate the main theorem of this paper:

Theorem 1.1. Let $k \geqslant k_{0}:=[n / 2 m]+6$ and $M>0$ be given. Assume that $F$ satisfies Assumption 2.1 and that $u^{0} \in H^{k m}(\Omega), u^{1} \in H^{(k-1) m}(\Omega), f \in \mathscr{C}_{T}^{k-2} \cap C^{k-1}\left([0, T], L_{2}(\Omega)\right)$ such that $\left(u^{0}, u^{1}, f\right) \in \mathscr{I}$ satisfies the natural compatibility condition of order $k$ (compare section 3). Then there exists a $T^{\prime} \in(0, T]$ depending only on

$$
\begin{aligned}
N:= & \left\|u^{0}\right\|_{k_{0} m}+\left\|u^{1}\right\|_{\left(k_{0}-1\right) m}+\int_{0}^{T}\left(\sum_{j=1}^{k_{0}-2}\left\|\partial_{i}^{j} f(t)\right\|_{\left(k_{0}-2-j\right) m}+\left\|\partial_{t}^{k_{0}-1} f(t)\right\|\right) \mathrm{d} t \\
& +\sup _{[0, T]}\left(\sum_{j=0}^{k_{0}-2}\left\|\partial_{t}^{j} f(t)\right\|_{\left(k_{0}-2-j\right) m}\right)
\end{aligned}
$$

(and $F, \Omega, M$ ) such that (1.1)-(1.3) has a unique solution $u \in \mathscr{C}_{T^{\prime}}^{k}$, which is a classical 
solution. If the assumptions are satisfied for every $k \geqslant k_{0}$, then

$$
u \in C^{\infty}\left(\left[0, T^{\prime}\right] \times \bar{\Omega}\right) \text {. }
$$

Remark (1) The regularity conditions made on $u^{0}$ and $u^{1}$ are necessary conditions for a solution $u$ to be in $\mathscr{C}_{T}^{k}$, as will be shown in section 3 . Therefore, we can continue the solution $u$ for $t>T^{\prime}$ by Theorem 1.1 as long as $\sup _{x \in \overline{\mathbf{\Omega}}}|\mathscr{D} u(t, x)| \leqslant \frac{1}{2} M$. If Assumption 2.1 is satisfied for every $M>0$, then we can continue the solution until a blow-up occurs.

Remark (2) In addition to Theorem 1.1 we prove that

$$
T^{\prime} \geqslant O\left(\log \log N^{-1}\right) \text { as } N \downarrow 0
$$

(compare section 7).

Remark (3) If problem (1.1)-(1.3) is quasilinear as in example 2 at the end of the section 2, it suffices to suppose that $k \geqslant k_{0}-1$ in Theorem 1.1. This can be shown by a modification of the proof of Lemma 6.1.

In the case $m=1, k \geqslant[n / 2]+8$, Theorem 1.1 has already been proved by Shibatah and Tsutsumi [7]. In addition to generalization of their result to higher values of $m$ we give a simpler proof, which makes possible the estimate (1.14).

The proof of Theorem 1.1 is based on the following idea: let $u \in \mathscr{C}_{T}^{k_{0}}$ be a solution of (1.1)-(1.3). Differentiating (1.1) with respect to $t$ we obtain (1.5). We set $v:=\partial_{\imath} u$ and conclude that

$$
\left.\begin{array}{ll}
\partial_{t}^{2} v(t)+\mathscr{A}_{u}(t) v(t)+\mathscr{B}_{u}(t) \partial_{t} v(t)=g_{u}(t) & \text { for } t \in[0, T], \\
v(t) \in \stackrel{\circ}{H}^{m}(\Omega) & \text { for } t \in[0, T], \\
v(0)=u^{1}, \quad \partial_{t} v(0)=u^{2}, & \text { for } t \in[0, T] \\
u(t)=u^{0}+\int_{0}^{t} v(\tau) \mathrm{d} \tau &
\end{array}\right\}
$$

Here we omit the variable $x$ and understand the equations in $L_{2}(\Omega)$. Then $(u, v)$ (sufficiently smooth) is a solution of (1.15) and (1.16) if and only if $v=\partial_{t} u$ and $u$ solves (1.1)-(1.3).

We define a mapping $\Phi: u \mapsto \Phi[u]$ by

$$
\Phi[u](t):=u^{0}+\int_{0}^{t} v(\tau) \mathrm{d} \tau \text { for } t \in[0, T],
$$

where $v$ is the unique solution of (1.15). By an existence theorem for linear equations contained in [6] it follows that $\Phi$ is a mapping from

$$
\tilde{\mathscr{C}}_{T}^{k}:=\bigcap_{j=1}^{k} C^{j}\left([0, T], H^{(k-j) m}(\Omega)\right)
$$

into itself. Note that $C\left([0, T], H^{k m}(\Omega)\right)$ does not contain $\tilde{\mathscr{C}}_{T}^{k}$, but $\mathscr{C}_{T}^{k}$. By energy estimates it follows that $\Phi$ is a contraction. This will give us the existence and uniqueness of a fixed point $u \in \tilde{\mathscr{C}}_{T}^{k_{0}}$ of $\Phi$ for some $T^{\prime}>0$, which is a solution of (1.1)-(1.3). The higher regularity of $u$ can be proved by induction in the same time interval $\left[0, T^{\prime}\right]$. 
This method could also be applied to hyperbolic systems and to hyperbolic equations with non-linear boundary conditions. In a conference in March 1990 the author learned that $\mathrm{H}$. Koch [5] uses the same idea to study equations of second order ( $m=1)$ with non-linear boundary conditions.

The author is thankful to Prof. Leis for giving him the opportunity of staying one and a half year in the stimulating atmosphere of his group.

This paper was partially supported by the Deutsche Forschungsgemeinschaft (SFB 256).

\section{Notation and assumptions}

By $\Omega$ we denote a domain in $\mathbb{R}^{n}$ having a smooth boundary $\partial \Omega \in C^{\infty}$ such that $\partial \Omega$ is bounded (or empty) or such that $\Omega$ has the form

$$
\Omega=\mathbb{R}^{n^{\prime}} \times \Omega^{\prime}
$$

with bounded $\Omega^{\prime} \subset \mathbb{R}^{n-n^{\prime}}$. The set $C_{0}^{\infty}(\Omega)$ consists of all the infinitely many differentiable functions having compact support in $\Omega$. Futhermore, let

$$
C_{b}^{j}(\Omega):=\left\{\varphi \in C^{j}(\Omega): \partial_{x}^{\alpha} \varphi \text { is bounded in } \Omega \text { for } \alpha \in \mathbb{N}_{0}^{n},|\alpha| \leqslant j\right\},
$$

where $|\alpha|:=\alpha_{1}+\cdots+\alpha_{n}$. For every sufficiently smooth function $\varphi$ we set

$$
D_{x}^{j} \varphi:=\left(\partial_{x}^{\alpha} \varphi: \alpha \in \mathbb{N}_{0}^{n},|\alpha|=j\right), \quad \bar{D}_{x}^{j} \varphi:=\left(\partial_{x}^{\alpha} \varphi: \alpha \in \mathbb{N}_{0}^{n},|\alpha| \leqslant j\right)
$$

$(j \in \mathbb{N})$. By $\langle.,$.$\rangle and \|\cdot\|$ we denote the inner product and the norm in $L_{2}(\Omega)$, and $\|\cdot\|_{j}$ denotes the norm in the $j$ th Sobolev space $H^{j}(\Omega)$.

If $u \in \mathscr{C}_{T}^{k}$ (compare (1.7)), then

$$
\begin{aligned}
|u(t)|_{k} & :=\sum_{j=0}^{k}\left\|\partial_{t}^{j} u(t)\right\|_{(k-j) m} \text { for } t \in[0, T], \\
\|\| u \mid \|_{k, T} & :=\sup _{[0, T]}|u(t)|_{k} .
\end{aligned}
$$

For $u \in \tilde{\mathscr{C}}_{T}^{k}$ (compare (1.18)) we set

$$
\begin{aligned}
|u(t)|_{k}^{\tilde{k}} & =\sum_{j=1}^{k}\left\|\partial_{t}^{j} u(t)\right\|_{(k-j) m}+\|u(t)\|_{(k-1) m} \\
& =\left|\partial_{t} u(t)\right|_{k-1}+\|u(t)\|_{(k-1) m} \text { for } t \in[0, T], \\
\|\| u\|\|_{\tilde{k}, T} & :=\sup _{[0, T]}|u(t)|_{k} .
\end{aligned}
$$

In order to pose the assumptions to be made on $F$, we set $\mu:=\left(\mu_{0}, \mu_{1}, \mu_{2}\right)$ with $\mu_{0}=\left(\mu_{0}^{(\alpha)}:|\alpha| \leqslant 2 m\right), \mu_{1}=\left(\mu_{1}^{(\beta)}:|\beta| \leqslant m\right)$ and $\mu_{0}^{(\alpha)}, \mu_{1}^{(\beta)}, \mu_{2} \in \mathbb{C}$. This notation means that in the case $\mu=\mathscr{D} u(t, x)$ we have $\mu_{0}^{(\alpha)}=\partial_{x}^{\alpha} u(t, x), \mu_{1}^{(\beta)}=\partial_{x}^{\beta} \partial_{t} u(t, x)$ and $\mu_{2}=\partial_{t}^{2} u(t, x)$. The operators $\mathscr{A}_{u}$ and $\mathscr{B}_{u}$ used in (1.5) and (1.15) are given by

$$
\begin{aligned}
& \mathscr{A}_{u}(t)=\sum_{|\alpha| \leqslant 2 m} a_{\alpha}(t, ., \mathscr{D} u(t, .)) \partial_{x}^{\alpha}, \\
& \mathscr{B}_{u}(t)=\sum_{|\beta| \leqslant m} b_{\beta}(t, ., \mathscr{D} u(t, .)) \partial_{x}^{\beta},
\end{aligned}
$$


where

$$
\begin{aligned}
& a_{\alpha}(t, x, \mu)=\left(\frac{\partial F}{\partial \mu_{2}}(t, x, \mu)\right)^{-1} \frac{\partial F}{\partial \mu_{0}^{(\alpha)}}(t, x, \mu), \\
& b_{\beta}(t, x, \mu)=\left(\frac{\partial F}{\partial \mu_{2}}(t, x, \mu)\right)^{-1} \frac{\partial F}{\partial \mu_{1}^{(\beta)}}(t, x, \mu) .
\end{aligned}
$$

Furthermore, $g_{u}$ used in (1.5) and (1.15) is given by

$$
\left.\begin{array}{rl}
g_{u}(t, x) & =g(t, x, \mathscr{D} u(t, x)), \\
g(t, x, \mu) & =\left(\frac{\partial F}{\partial \mu_{2}}(t, x, \mu)\right)^{-1}\left[\partial_{t} f(t, x)-\left(\partial_{t} F\right)(t, x, \mu)\right] .
\end{array}\right\}
$$

Note that $\mathscr{D} u(t, x)$ has

$$
q(n, m):=\left(\begin{array}{c}
2 m+n \\
n
\end{array}\right)+\left(\begin{array}{c}
m+n \\
n
\end{array}\right)+1
$$

components. We impose the following conditions on $F$.

Assumption 2.1. Let $k \in \mathbb{N}$ and $M, T>0$ be given. Then

(i) $F \in C_{b}^{(k-2) m+1}\left([0, T] \times \bar{\Omega} \times\left\{\mu \in \mathbb{R}^{q(n, m)}:|\mu| \leqslant M\right\}\right)$.

(ii) $F(t, x, 0)=0$ in $[0, T] \times \overline{\mathbf{\Omega}}$.

(iii) $\partial F(t, x, \mu) / \partial \mu_{2}$ is real valued and

$$
\frac{\partial F}{\partial \mu_{2}}(t, x, \mu) \geqslant d_{1}>0 \text { on }[0, T] \times \bar{\Omega} \times\{\mu:|\mu| \leqslant M\} \text {. }
$$

(iv) There exists a $d_{2}>0$ such that

$$
(-1)^{m} \operatorname{Re} \sum_{|\alpha|=2 m} a_{a}(t, x, \mu) \xi^{\alpha} \geqslant d_{2}|\xi|^{2 m} \quad \text { on }[0, T] \times \bar{\Omega} \times\{\mu:|\mu| \leqslant M\}
$$

for every $\xi \in \mathbb{R}^{n}\left(\xi^{\alpha}:=\xi_{1}^{\alpha_{1}} \ldots \xi_{n}^{\alpha_{n}}\right)$. Furthermore, for every $u \in \mathscr{C}_{T}^{k_{1}}\left(k_{1}:=[n / 2 m]\right.$ $+4)$ there exists a $d_{3}>0$, which may depend on $\|u\| \|_{k_{1}, r}$, such that

$$
\left.\| \mathscr{A}_{u}(t)-\mathscr{A}_{u}^{*}(t)\right) \varphi\left\|\leqslant d_{3}\right\| \varphi \|_{m} \text { for } \varphi \in H^{2 m}(\Omega) \cap \dot{H}^{m}(\Omega), t \in[0, T]
$$

here $\mathscr{A}_{u}^{*}(t)$ denotes the adjoint operator to $\mathscr{A}_{u}(t)$ with $D\left(\mathscr{A}_{u}(t)\right)=H^{2 m}(\Omega)$ $\cap \dot{H}^{m}(\Omega)$.

(v) For every $u \in \mathscr{C}_{T}^{k_{1}}$ there exists a $d_{4} \in \mathbb{R}$ (not necessarily positive), which may depend on $\|u \mid\|_{k_{1}, T}$, such that

$$
\operatorname{Re}\left\langle\mathscr{B}_{u}(t) \varphi, \varphi\right\rangle \geqslant d_{4}\|\varphi\|^{2} \text { for } \varphi \in \stackrel{\circ}{H}^{m}(\Omega), \quad t \in[0, T] .
$$

Condition (2.13) means that the part of $\mathscr{A}_{u}$ containing the derivatives $\partial_{x}^{a}$ with $m+1 \leqslant|\alpha| \leqslant 2 m$ is symmetric. An analogous condition was used in [2].

Condition (2.14) seems to be complicated. However, in the case $m=1$ this condition is satisfied for every real-valued $F$, since

$$
\begin{aligned}
& \sum_{|\beta|=1}\left\langle b_{\beta}(t, ., \mathscr{D} u(t)) \partial_{x}^{\beta} \varphi, \varphi\right\rangle=-\sum_{|\beta|=1}\left\langle\varphi, \partial_{x}^{\beta}\left[b_{\beta}(t, ., \mathscr{D} u(t)) \varphi\right]\right\rangle \\
& =-\sum_{|\beta|=1}\left\langle\varphi, b_{\beta}(t, ., \mathscr{D} u(t)) \partial_{x}^{\beta} \varphi\right\rangle-\sum_{|\beta|=1}\left\langle\varphi,\left[\partial_{x}^{\beta} b_{\beta}(t, ., \mathscr{D} u(t))\right] \varphi\right\rangle
\end{aligned}
$$


and therefore

$$
\begin{aligned}
\operatorname{Re}\left\langle\mathscr{B}_{u}(t) \varphi, \varphi\right\rangle & =-\frac{1}{2} \sum_{|\beta|=1}\left\langle\varphi,\left[\partial_{x}^{\beta} b_{\beta}(t, ., \mathscr{D} u(t))\right] \varphi\right\rangle+\left\langle b_{0}(t, ., \mathscr{D} u(t)) \varphi, \varphi\right\rangle \\
& \geqslant c\|\varphi\|^{2} .
\end{aligned}
$$

In the same way it follows in the case $m=2$ that (2.14) holds if $F$ is real-valued and satisfies

$$
-\sum_{|\beta|=2} b_{\beta}(t, x, \mu) \xi^{\beta} \geqslant 0 \text { on }[0, T] \times \bar{\Omega} \times\{\mu:|\mu| \leqslant M\}
$$

for every $\xi \in \mathbb{R}^{n}$. If $m \in \mathbb{N}$ and $\mathscr{B}_{u}(t)$ is elliptic for every $t \in[0, T]$, then (2.14) also holds. Example 1. Let $F$ be defined by (1.4). Then Assumption 2.1 is satisfied if

$$
a_{\alpha} \in C_{b}^{(k-2) m+1}\left([0, T] \times \bar{\Omega} \times\left\{\mu_{0}:\left|\mu_{0}\right| \leqslant M\right\}\right)
$$

is real valued for $|\alpha|=2$ and

$$
-\operatorname{Re} \sum_{|\alpha|=2}\left(a_{\alpha}\left(t, x, \mu_{0}\right)+\sum_{|\gamma| \leqslant 2} \frac{\partial a_{\gamma}\left(t, x, \mu_{0}\right)}{\partial \mu_{0}^{(\alpha)}} \mu_{0}^{(\gamma)}\right) \xi^{\alpha} \geqslant d_{2}|\xi|^{2 m}
$$

on $[0, T] \times \bar{\Omega} \times\left\{\mu_{0}:\left|\mu_{0}\right| \leqslant M\right\}$ for every $\xi \in \mathbb{R}^{n}$.

Example 2. For the quasilinear equation let

$$
\begin{aligned}
F(t, x, \mathscr{D} u(t, x)):= & \partial_{t}^{2} u(t, x) \\
& +\sum_{|\alpha|,|\beta| \leqslant m}(-1)^{|\alpha|} \partial_{x}^{\alpha}\left(a_{\alpha \beta}\left(t, x, \bar{D}_{x}^{m} u(t, x), \partial_{t} u(t, x)\right) \partial_{x}^{\beta}(t, x)\right) \\
& +h\left(t, x, \overline{D_{x}^{m}} u(t, x), \partial_{t} u(t, x)\right)
\end{aligned}
$$

with $a_{\alpha \beta}, h \in C_{b}^{(k-1) m+1}\left([0, T] \times \bar{\Omega} \times\left\{\left(\mu_{1}, \mu_{2}\right):\left|\mu_{1}\right|+\left|\mu_{2}\right| \leqslant M\right\}\right), a_{\alpha \beta}=\bar{a}_{\beta \alpha}$ for $|\alpha|$ $+|\beta|>m, h(t, x, 0)=0$ on $[0, T] \times \bar{\Omega}$ and

$$
\operatorname{Re} \sum_{|\alpha| \cdot|\beta|=m} a_{a \beta}\left(t, x, \mu_{1}, \mu_{2}\right) \xi^{\alpha+\beta} \geqslant d_{2}|\xi|^{2 m}
$$

on $[0, T] \times \bar{\Omega} \times\left\{\left(\mu_{1}, \mu_{2}\right):\left|\mu_{1}\right|+\left|\mu_{2}\right| \leqslant M\right\}$ for every $\xi \in \mathbb{R}^{n}$, then Assumption 2.1 is satisfied.

\section{The compatibility condition}

Let $\left(u^{0}, u^{1}, f\right) \in \mathscr{I}$ be given and let $u \in \mathscr{C}_{T}^{k}$ with $k \geqslant[n / 2 m]+3$ be a solution of (1.1)-(1.3). Then it holds that $u(t) \in \mathscr{H}^{m}(\Omega)$ and $u \in C^{k-1}\left([0, T], H^{m}(\Omega)\right)$. Note that this means that there exist functions $\partial_{i}^{j} u \in C\left([0, T], H^{m}(\Omega)\right)$ such that

$$
\left\|\partial_{t}^{j} u(t)-\frac{\partial_{t}^{j-1} u(t+h)-\partial_{t}^{j-1} u(t)}{h}\right\|_{m} \rightarrow 0 \quad \text { as } h \rightarrow 0
$$

for $0<t<T, j=1, \ldots, k-1$. If $t=0$ or $t=T$, (3.1) must hold as $h \downarrow 0$ or $h \uparrow 0$, respectively.

Since $\dot{H}^{m}(\Omega)$ is closed in $H^{m}(\Omega)$, we conclude from $u(t) \in \dot{H}^{m}(\Omega)$ and (3.1) that $\partial_{i}^{j} u(t) \in \stackrel{\circ}{H}^{m}(\Omega)$ for $t \in[0, T], j=0, \ldots, k-1$. In particular, we have

$$
\partial_{t}^{j} u(0) \in \stackrel{H}{H}^{m}(\Omega) \text { for } j=0, \ldots, k-1 \text {. }
$$


In order to formulate the compatibility condition, we note that $\partial_{l}^{j} u(0)=u^{j}$ for $j=0,1,2$, where $u^{2}$ is given by $\left(u^{0}, u^{1}, f\right) \in \mathscr{I}$ and (1.10) and (1.11); the fact that $\partial_{t}^{2} u(0)=u^{2}$ will be proved by Lemma 3.1 below. We express $\partial_{t}^{j} u(0)$ for $j \geqslant 3$ in terms of $u^{0}, u^{1}, u^{2}$ and $f$. Differentiating (1.1) with respect to $t$ we obtain

$$
\partial_{t}^{3} u(t)=g_{u}(t)-\mathscr{A}_{u}(t) \partial_{t} u(t)-\mathscr{B}_{u}(t) \partial_{t}^{2} u(t) \text { for } t \in[0, T]
$$

(compare (1.5), (2.7)-(2.11)). Further differentiation yields

$$
\begin{aligned}
\partial_{t}^{j} u(t)= & \partial_{t}^{j-3} g_{u}(t)-\sum_{v=0}^{j-3}\left(\begin{array}{c}
j-3 \\
v
\end{array}\right) \\
& \times\left\{\left[\partial_{t}^{v} \mathscr{A}_{u}(t)\right] \partial_{t}^{j-2-v} u(t)+\left[\partial_{t}^{v} \mathscr{B}_{u}(t)\right] \partial_{t}^{j-1-v} u(t)\right\}
\end{aligned}
$$

$(j \geqslant 3)$, where

$$
\partial_{t}^{v} \mathscr{A}_{u}(t):=\sum_{|\alpha| \leqslant 2 m}\left[\partial_{t}^{v} a_{\alpha}(t, ., \mathscr{D} u(t))\right] \partial_{x}^{\alpha}
$$

and $\partial_{t}^{v} \mathscr{B}_{u}(t)$ is defined analogously. We set recursively

$$
\begin{aligned}
u^{j}:= & \partial_{t}^{j-3} g_{u}(0)-\sum_{v=0}^{j-3}\left(\begin{array}{c}
j-3 \\
v
\end{array}\right) \\
& \times\left\{\left[\partial_{t}^{v} \mathscr{A}_{u}(0)\right] u^{j-2-v}+\left[\partial_{t}^{v} \mathscr{B}_{u}(0)\right] u^{j-1-v}\right\}
\end{aligned}
$$

for $j=3, \ldots, k$, where we have set $\partial_{t}^{\mu} u(0):=u^{\mu}$ for $\mu=0, \ldots, j-1$ in the coefficients of $\partial_{t}^{v} \mathscr{A}_{u}(0)$ and $\partial_{t}^{v} \mathscr{B}_{u}(0)$. Note that $u^{j}=\partial_{i}^{j} u(0)$ for $j=0, \ldots, k$ from our considerations.

Definition 3.1. Let $\left(u^{0}, u^{1}, f\right) \in \mathscr{I}$ be given. We say that $\left(u^{0}, u^{1}, f\right)$ satisfies the compatibility condition of order $k \in \mathbb{N}$, if $u^{j} \in \stackrel{H}{H}^{m}(\Omega)$ for $j=0, \ldots, k-1$, where $u^{2}$ is given by (1.10) and (1.11), and $u^{j}$ is defined by (3.4) for $j \geqslant 3$.

Now we show that the solution $u^{2}$ of $(1.10)$ and $(1.11)$ is unique.

Lemma 3.1. Assume that $u^{0} \in C^{2 m}(\bar{\Omega}), u^{1} \in C^{m}(\bar{\Omega}), f(0) \in C(\bar{\Omega})$ and that $F$ satisfies Assumption 2.1 for some $k \geqslant 2$. Let $x \in \bar{\Omega}$ be fixed. If $y \in \mathbb{C}$ solves

$$
\begin{aligned}
& F\left(0, x, \bar{D}_{x}^{2 m} u^{0}(x), \bar{D}_{x}^{m} u^{1}(x), y\right)=f(0, x), \\
& \left|\bar{D}_{x}^{2 m} u^{0}(x)\right|+\left|\bar{D}_{x}^{m} u^{1}(x)\right|+|y| \leqslant M,
\end{aligned}
$$

then $y$ is unique.

Proof. This proof is the same as a proof in [7]. Let $y_{1}, y_{2} \in \mathbb{C}$ be two solutions of (3.5) and (3.6). Then it holds that

$$
\left|\bar{D}_{x}^{2 m} u^{0}(x)\right|+\left|\bar{D}_{x}^{m} u^{1}(x)\right|+\left|y_{1}+\Theta\left(y_{2}-y_{1}\right)\right| \leqslant M
$$

for $0 \leqslant \Theta \leqslant 1$. Furthermore, we have

$$
\begin{aligned}
0 & =F\left(0, x, \bar{D}_{x}^{2 m} u^{0}(x), \bar{D}_{x}^{m} u^{1}(x), y_{1}\right)-F\left(0, x, \bar{D}_{x}^{2 m} u^{0}(x), \bar{D}_{x}^{m} u^{1}(x), y_{2}\right) \\
& =-\int_{0}^{1} \frac{\partial F}{\partial \mu_{2}}\left(0, x, \bar{D}_{x}^{2 m} u^{0}(x), \bar{D}_{x}^{m} u^{1}(x), y_{1}+\Theta\left(y_{2}-y_{1}\right)\right)\left(y_{2}-y_{1}\right) \mathrm{d} \Theta
\end{aligned}
$$

Since $\partial F(0, x, \mu) / \partial \mu_{2}>0$, this implies that $y_{2}=y_{1}$.

Our considerations yield the following lemma. 
Lemma 3.2. Let $k \geqslant[n / 2 m]+3$ and $M>0$ be given. Suppose that $F$ satisfies Assumption 2.1 and that $u^{0} \in H^{k m}(\Omega), u^{1} \in H^{(k-1) m}(\Omega), f \in \mathscr{C}_{T}^{k-2}$ such that $\left(u^{0}, u^{1}, f\right) \in \mathscr{I}$. If $u \in \mathscr{C}_{T}^{k}$ is a solution of (1.1)-(1.3) with the property (1.9), then it holds that $\partial_{i}^{j} u(0)=u^{j}$, where $u^{2}$ is determined by (1.10)-(1.11) and $u^{j}$ is given by (3.4) for $j=3, \ldots, k$, Furthermore, $\left(u^{0}, u^{1}, f\right)$ satisfies the compatibility condition of order $k$.

Let $u \in \mathscr{C}_{T}^{k}(k \geqslant[n / 2 m]+3)$ be a solution of (1.1)-(1.3). According to Lemma 3.2 it holds that

$$
|u(0)|_{k}=\left\|u^{0}\right\|_{(k-1) m}+\sum_{j=1}^{k}\left\|u^{j}\right\|_{(k-j) m}
$$

We prove an estimate for $|u(0)| \tilde{k}$.

Lemma 3.3. Let $k \geqslant[n / 2 m]+4$ and $R>0$ be given and let all assumptions of Lemma 3.2 be satisfied. Furthermore, suppose that

$$
\left\|u^{0}\right\|_{k m}+\left\|u^{1}\right\|_{(k-1) m}+|f(0)|_{k-2} \leqslant R .
$$

Then there exists a positive number $c=c(R, k)$ such that

$$
\left\|u^{j}\right\|_{(k-j) m} \leqslant c\left(\left\|u^{0}\right\|_{k m}+\left\|u^{1}\right\|_{(k-1) m}+|f(0)|_{k-2}\right)
$$

for $j=2, \ldots, k$.

Proof. At first we prove (3.8) for $j=2$. We set

$$
B:=\sup \left\{\left|\nabla_{\mu} F(0, x, \mu)\right|: x \in \bar{\Omega},|\mu| \leqslant M\right\},
$$

where $\nabla_{\mu} F:=\left(\partial F / \partial \mu_{0}^{(\alpha)}, \partial F / \partial \mu_{1}^{(\beta)}, \partial F / \partial \mu_{2}:|\alpha| \leqslant 2 m,|\beta| \leqslant m\right)$. Furthermore, we set $\mathscr{D} u(0, x):=\left(\bar{D}_{x}^{2 m} u^{0}(x), \bar{D}_{x}^{m} u^{1}(x), u^{2}(x)\right)$. Let $x \in \Omega$ be fixed. From $F(0, x, 0)=0$ and (1.10) we conclude that

$$
\begin{aligned}
f(0, x) & =F(0, x, \mathscr{D} u(0, x))-F(0, x, 0) \\
& =\nabla_{\mu} F(0, x, \Theta \mathscr{D} u(0, x)) \cdot\left(\bar{D}_{x}^{2 m} u^{0}(x), \bar{D}_{x}^{m} u^{1}(x), u^{2}(x)\right)
\end{aligned}
$$

for some $\Theta \in(0,1)$. Note that $\left(\partial F / \partial \mu_{2}\right)(t, x, \mu) \geqslant d_{1}>0$ by Assumption 2.1. Hence we obtain from (3.9) and (3.10) that

$$
\left|u^{2}(x)\right| \leqslant d_{1}^{-1}\left[|f(0, x)|+B\left(\left|\bar{D}_{x}^{2 m} u^{0}(x)\right|+\left|\bar{D}_{x}^{m} u^{1}(x)\right|\right)\right]
$$

for $x \in \Omega$. This implies that

$$
\left\|u^{2}\right\| \leqslant c\left(\|f(0)\|+\left\|u^{0}\right\|_{2 m}+\left\|u^{1}\right\|_{m}\right) \text {. }
$$

We differentiate (1.10) with respect to $x_{i}$ and divide the result by $\partial F / \partial \mu_{2}$. This yields

$$
\begin{aligned}
\frac{\partial u^{2}(x)}{\partial x_{i}}= & -\mathscr{A}_{u}(0) \frac{\partial u^{0}(x)}{\partial x_{i}}-\mathscr{A}_{u}(0) \frac{\partial u^{1}(x)}{\partial x_{i}} \\
& +\left(\frac{\partial F}{\partial \mu_{2}}(0, x, \mathscr{D} u(0, x))\right)^{-1}\left(\frac{\partial f(0, x)}{\partial x_{i}}-\frac{\partial F}{\partial x_{i}}(0, x, \mathscr{D} u(0, x))\right)
\end{aligned}
$$

for $x \in \Omega, i=1, \ldots, n$. It follows by induction that

$$
\begin{aligned}
\partial_{x}^{\alpha} u^{2}(x)= & \sum_{\gamma} h_{\gamma}^{(\alpha)}(0, x, \mathscr{D} u(0, x)) \prod_{j=1}^{|\alpha|}\left[D_{x}^{j}\left(\bar{D}_{x}^{2 m} u^{0}(x), \bar{D}_{x}^{m} u^{1}(x), f(0, x)\right)\right]^{\gamma_{j}} \\
& -\left(\frac{\partial F(0, x, \mathscr{D} u(0, x))}{\partial \mu_{2}}\right)^{-1}\left(\partial_{x}^{\alpha} F\right)(0, x, \mathscr{D} u(0, x))
\end{aligned}
$$


for $x \in \Omega,|\alpha| \leqslant(k-2) m$, where the summation extends over all $\gamma=\left(\gamma_{1}, \ldots, \gamma_{|\alpha|}\right)$ with

$$
1 \leqslant \sum_{j=1}^{|a|} j\left|\gamma_{j}\right| \leqslant|\alpha|
$$

and functions $h_{y}^{(\alpha)}$ consist of derivatives of $F$. Note that $(k-2) m \geqslant[n / 2]+2$ and that $\partial_{x}^{\alpha} F(0, x, 0)=0$ on $\bar{\Omega}$. With Corollary A.4 and Lemma A.6 we obtain that

$$
\left\|\partial_{x}^{a} u^{2}\right\| \leqslant c\left(\left\|u^{0}\right\|_{k m}+\left\|u^{1}\right\|_{(k-1) m}+\|f(0)\|_{(k-2) m}\right)
$$

for $|\alpha| \leqslant(k-2) m$. This together with (3.12) proves (3.8) for $j=2$.

In order to prove (3.8) for $j \geqslant 3$ we rewrite (3.3) for $t=0$ as

$$
\begin{aligned}
\partial_{t}^{j} u(0, x)= & \sum_{\gamma} \tilde{h}_{\gamma}^{(j)}(0, x, \mathscr{D} u(0, x)) \prod_{i=1}^{j-2}\left(\bar{D}_{x}^{2 m} u^{i}(x), \bar{D}_{x}^{m} u^{i+1}(x), \partial_{\imath}^{i} f(0, x)\right)^{\gamma_{i}} \\
& -\left(\frac{\partial F(0, x, \mathscr{D} u(0, x))}{\partial \mu_{2}}\right)^{-1}\left(\partial_{t}^{j-2} F\right)(0, x, \mathscr{D} u(0, x))
\end{aligned}
$$

$(j=3, \ldots, k)$; here the summation extends over all $\gamma=\left(\gamma_{1}, \ldots, \gamma_{j}\right)$ with

$$
1 \leqslant \sum_{i=1}^{j-2} i\left|\gamma_{i}\right| \leqslant j-2
$$

and the functions $\tilde{h}_{\gamma}^{(j)}$ consist of derivatives of $F$. In order to apply Lemma A.6 to the product in (3.15) we have to set

$$
w_{r}:= \begin{cases}\left(\bar{D}_{x}^{2 m} u^{r / m}, \bar{D}_{x}^{m} u^{r / m+1}, \partial_{t}^{r / m} f(0)\right) & \text { for } r=i m, i=1, \ldots, j-2, \\ 0 & \text { otherwise. }\end{cases}
$$

Then we obtain from (3.15) by Corollary A.4, Lemma A.6 and Lemma A.2 (for functions that are constant in $t$ ) that

$$
\left\|u^{j}\right\|_{(k-j) m} \leqslant c\left(\sum_{i=1}^{j-1}\left\|u^{i}\right\|_{(k-i) m}+|f(0)|_{k-2}\right) .
$$

From this (3.8) for $j=3, \ldots, k$ follows by induction.

We conclude this section by proving the existence of data $\left(u^{0}, u^{1}, f\right) \in \mathscr{I}$ satisfying the compatibility condition of order $k$.

Lemma 3.4. Let $k \geqslant[n / 2 m]+3$ and $M>0$ be given. Suppose that $F$ satisfies Assumption 2.1 and that $u^{0} \in C_{0}^{k m}(\Omega), u^{1} \in C_{0}^{(k-1) m}(\Omega), f \in C^{(k-2) m}([0, T] \times \Omega)$ such that $f(0) \in C_{0}^{(k-2) m}(\Omega)$ and

$$
\sup _{x \in \overline{\mathbf{\Omega}}}\left[\frac{1}{d_{1}}|f(0, x)|+\left(\frac{B}{d_{1}}+1\right)\left(\left|\bar{D}_{x}^{2 m} u^{0}(x)\right|+\left|\bar{D}_{x}^{m} u^{1}(x)\right|\right)\right] \leqslant \frac{1}{2} M,
$$

where $B$ is defined by (3.9) and $d_{1}$ is given by Assumption 2.1. Then it holds that $\left(u^{0}, u^{1}, f\right) \in \mathscr{I}$ and $u^{j} \in C_{0}^{(k-j) m}(\Omega) \subset \dot{H}^{m}(\Omega)$ for $j=2, \ldots, k-1$. In particular, $\left(u^{0}, u^{1}, f\right)$ satisfies the compatibility condition of order $k$.

\section{Proof. Let}

$$
S:=\{x \in \Omega \text { : there exists a } y=y(x) \in \mathbb{C} \text { such that (3.5) and (3.6) hold }\} .
$$

Note that $S^{\prime}:=\left\{x \in \Omega: u^{0}(x)=u^{1}(x)=f(0, x)=0\right\} \subset S$, since $F(0, x, 0)=0$ and therefore $y(x)=0$ on $S^{\prime}$. 
By the implicit function theorem it follows that $S$ is an open subset of $\Omega$ with respect to the usual metric on $\Omega \subset \mathbb{R}^{n}$. In fact, if $x \in S$, then $\left(\partial F / \partial \mu_{2}\right)(0, x, \mu) \geqslant d_{1}>0$ and the implicit function theorem implies the existence of a neighbourhood $U$ of $x$ and a solution $y \in C(U)$ of (3.5). By (3.11) and (3.17) we obtain that $\left|\bar{D}_{x}^{2 m} u^{0}(x)\right|$ $+\left|\bar{D}_{x}^{m} u^{1}(x)\right|+|y(x)| \leqslant \frac{1}{2} M$ on $U$. Hence we have $U \subset S$. Moreover, we obtain that $y \in C(S)$, since the solution $y$ of (3.5) and (3.6) is unique by Lemma 3.1 and since for every $x \in S$ there exists such a neighbourhood $U$ with $y \in C(U)$.

On the other hand, since $y \in C(S)$, it follows from the continuity assumptions made on $F, u^{0}, u^{1}$ and $f$ that $S$ is a closed subset of $\Omega$. Thus $S=\Omega$ and $y \in C(\Omega)$. Since $S^{\prime} \subset S$, we have $y \in C_{0}(\Omega)$. Setting $u^{2}:=y$ we obtain $\left(u^{0}, u^{1}, f\right) \in \mathscr{I}$. Using (3.13) and (3.15) we conclude that $u^{j} \in C_{0}^{(k-j) m}(\Omega)$ for $j=2, \ldots, k$. Hence Lemma 3.4 is proved.

\section{The existence of the fixed point of $\Phi$}

In this section we study the mapping $\Phi$, which is defined by (1.17) and (1.15). First we prove the following Lemma.

Lemma 4.1. Let $k \geqslant[n / 2 m]+5$ and $M>0$ be given. Suppose that $F$ satisfies Assumption 2.1 and that $u^{0} \in H^{k m}(\Omega), u^{1} \in H^{(k-1) m}(\Omega), f \in \mathscr{C}_{T}^{k-2} \cap C^{k-1}\left([0, T], L_{2}(\Omega)\right)$ such that $\left(u^{0}, u^{1}, f\right) \in \mathscr{I}$ satisfies the compatibility condition of order $k$ (compare Definitions 1.1 and 3.1). Then for every sufficiently large $R>0$ there exists a $T_{1}>0$ such that $\Phi$ maps

$$
\mathscr{M}\left(R, T_{1}\right):=\left\{u \in \tilde{\mathscr{C}}_{T_{1}}^{k}:\|u\| \tilde{r}_{k, T_{1}} \leqslant R, \partial_{t}^{j} u(0)=u^{j}(j=0, \ldots, k-1)\right\}
$$

into itself, where $u^{j}$ is given by (1.10), (1.11) and (3.4) for $j \geqslant 2$. Here $T_{1}$ depends only on $R, k$ and

$$
\tilde{N}_{k}=|u(0)|_{k}+\int_{0}^{T}\left|\left(\left.\partial_{1} f(\tau)\right|_{k-3}+\left\|\partial_{t}^{k-1} f(\tau)\right\|\right) \mathrm{d} \tau+\|\| \partial_{t} f \|\right|_{k-3, T}
$$

where

$$
|u(0)|_{k}=\sum_{j=1}^{k}\left\|u^{j}\right\|_{(k-j) m}+\left\|u^{0}\right\|_{(k-1) m} .
$$

Proof. We suppose that $R, T_{1}>0$ are arbitrary, but fixed. Let $u \in \mathscr{M}\left(R, T_{1}\right)$ be given. In a first step we show that $u$ satisfies (1.9) for sufficiently small $T_{1}$.

By Sobolev's lemma we obtain for $(t, x) \in\left[0, T_{1}\right] \times \bar{\Omega}$ that

$$
|\mathscr{D} u(t, x)-\mathscr{D} u(0, x)|=\left|\partial_{t} \mathscr{D} u(\Theta t, x)\right| t \leqslant c_{1} \||u| \mid \tilde{i}_{, T_{1}} t \leqslant c_{1} R T_{1} .
$$

Since $|\mathscr{D} u(0, x)| \leqslant \frac{1}{2} M$ for $x \in \bar{\Omega}$ by (1.11), it follows that $|\mathscr{D} u(t, x)| \leqslant M$ for $(t, x) \in\left[0, T_{1}\right] \times \bar{\Omega}$ if $T_{1} \leqslant M / 2 c_{1} R$.

In the next step we prove $\Phi[u] \in \tilde{\mathscr{C}}_{T_{1}}^{k}$. Consider (1.15). Formally differentiating this equation $j-2$ times with respect to $t$ and comparing the result with (3.4) we conclude that (formally)

$$
\partial_{t}^{j} v(0)=u^{j+1} \in \stackrel{\circ}{H}^{m}(\Omega), \quad j=0, \ldots, k-2 .
$$

Hence $\left(u^{1}, u^{2}, g_{u}\right)$ satisfies the natural compatibility condition for problem (1.15) of order $k-1$. From (2.9) and Lemma A.1 we obtain

$$
a_{\alpha}(., ., \mathscr{D} u)-a_{\alpha}(., ., 0) \in \tilde{\mathscr{C}}_{T_{1}}^{k-2} \text {. }
$$


The same holds for $b_{\beta}$. From (2.11) we obtain by Lemma A.1 and Corollary A.5 that

$$
g_{u} \in \tilde{\mathscr{C}}_{T_{1}}^{k-2} \subset \mathscr{C}_{T_{1}}^{k-3} \cap \mathscr{C}^{k-2}\left(\left[0, T_{1}\right], L_{2}(\Omega)\right) \text {. }
$$

From the linear existence theorem ((1.1) in [6]) it follows that (1.15) has a unique solution $v \in \mathscr{C}_{T_{1}}^{k-1}$. Therefore (4.4) holds. Furthermore we conclude from (1.17) and $v \in \mathscr{C}_{T_{1}}^{k-1}$ that $\Phi[u] \in C\left(\left[0, T_{1}\right], H^{(k-1) m}(\Omega)\right), \partial_{t} \Phi[u]=v \in \mathscr{C}_{T_{1}}^{k-1}$. This proves that $\Phi[u] \in \tilde{\mathscr{C}}_{T_{1}}^{k}$. Note that we have

$$
\partial_{i}^{j} \Phi[u](0)=u^{j} \quad \text { for } j=0, \ldots, k-1
$$

by (1.17) and (4.4).

In the next step we prove $\Phi[u] \in \mathscr{M}\left(R, T_{1}\right)$. We suppose that $T_{1} \leqslant 1, T_{1} \leqslant 1 / R$. By $d_{j}(j=1,2, \ldots)$ we denote positive constants depending only on $k$ and $\tilde{N}_{k}$. It holds that

$$
|u(t)|_{k-1} \leqslant|u(0)|_{k-1}+\int_{0}^{t}\left|\partial_{t} u(\tau)\right|_{k-1} \mathrm{~d} \tau \leqslant \tilde{N}_{k}+\int_{0}^{T_{1}} R \mathrm{~d} \tau \leqslant d_{1}
$$

for $t \in\left[0, T_{1}\right]$. With Lemma 8.1 we obtain that

$$
\begin{aligned}
& \left|a_{\alpha}(t, ., \mathscr{D} u(t))-a_{\alpha}(t, ., 0)\right|_{k-3} \leqslant d_{2}, \\
& \left|a_{\alpha}(t, ., \mathscr{D} u(t))-a_{\alpha}(t, ., 0)\right|_{k-2} \leqslant d_{3} R
\end{aligned}
$$

for $t \in\left[0, T_{1}\right]$. The same estimates hold for $b_{\beta}$. We apply Theorem 1.1 of [6] to (1.15). This yields

$$
\begin{aligned}
|v(t)|_{k-1} \leqslant & d_{4} \exp \left(c_{2} t\right)\left[|v(0)|_{k-1}+c_{2} \int_{0}^{t}\left(\left\|\partial_{t}^{k-2} g_{u}(\tau)\right\|+\left|g_{u}(\tau)\right|_{k-3}\right) \mathrm{d} \tau\right] \\
& +d_{5}\left|g_{u}(t)\right|_{k-3}
\end{aligned}
$$

for $t \in\left[0, T_{1}\right]$, where $c_{2}=c_{2}(R)$. Note that

$$
|v(0)|_{k-1}=\left|\partial_{t} u(0)\right|_{k-1} \leqslant \tilde{N}_{k}
$$

and that by Lemma A.1 and Corollary A.5

$$
\begin{gathered}
\left|g_{u}(t)\right|_{k-3} \leqslant d_{6}, \\
\int_{0}^{t}\left(\left\|\partial_{t}^{k-2} g_{u}(\tau)\right\|+\left|g_{u}(\tau)\right|_{k-3}\right) \mathrm{d} \tau \leqslant d_{7} T_{1}+d_{8} R T_{1}
\end{gathered}
$$

for $t \in\left[0, T_{1}\right]$. Hence it follows from (4.9) that

$$
|v(t)|_{k-1} \leqslant d_{9}+d_{10} \exp \left(c_{2} t\right)\left(1+c_{2} T_{1}(1+R)\right)
$$

for $t \in\left[0, T_{1}\right]$. With (1.15) we conclude that

$$
\begin{aligned}
\mid \Phi[u](t) \tilde{\mid}_{k} & \leqslant\left\|u^{0}\right\|_{(k-1) m}+\int_{0}^{t}\|v(\tau)\|_{(k-1) m} \mathrm{~d} \tau+|v(t)|_{k-1} \\
& \leqslant d_{11}+d_{10} \exp \left(c_{2} t\right)\left(1+\frac{1}{c_{2}}\right)\left(1+c_{2} T_{1}(1+R)\right)
\end{aligned}
$$

for $t \in\left[0, T_{1}\right]$. Note that $c_{2}$ in (4.9) can be chosen so that $c_{2} \geqslant 1$. Furthermore, note that $d_{10}$ and $d_{11}$ depend only on $k$ and $\tilde{N}_{k}$. We suppose that

$$
R \geqslant d_{11}+4 d_{10} e,
$$




$$
T_{1}:=\min \left\{T, 1, \frac{1}{R}, \frac{M}{2 c_{1} R}, \frac{1}{c_{2}(1+R)}\right\} .
$$

Then (4.10) implies that $\|\Phi[u]\| \tilde{r}_{k, T_{1}} \leqslant R$. This together with (4.5) proves that $\Phi[u] \in \mathscr{M}\left(R, T_{1}\right)$.

The next lemma contains the contraction property of $\Phi$.

Lemma 4.2. Let all assumptions of Lemma 4.1 be satisfied. Furthermore let $R>0$ be given. Then there exists a $T_{2}>0$ such that

$$
\left\|\Phi\left[u_{1}\right]-\Phi\left[u_{2}\right]\right\| \tilde{k}_{k-1, T_{2}} \leqslant \frac{1}{2}\left\|u_{1}-u_{2}\right\| \tilde{r}_{k-1, T_{2}}
$$

for all $u_{1}, u_{2} \in \mathscr{M}\left(R, T_{2}\right)$ with $\Phi\left[u_{1}\right], \Phi\left[u_{2}\right] \in \mathscr{M}\left(R, T_{2}\right)$. Here $T_{2}$ depends only on $R, k$ and $\left\|\partial_{t} f\right\|_{k}-3, T$.

Remark. If $u \in \mathscr{M}\left(R, T_{2}\right)$, then $\Phi[u] \in \mathscr{M}\left(R, T_{2}\right)$ if and only if $\|\Phi[u]\| \|_{k, T_{2}} \leqslant R$.

Proof of Lemma 4.2. We suppose that $T_{2}>0$ is arbitrary, but fixed. We set $v_{j}:=\partial_{t} \Phi\left[u_{j}\right](j=1,2)$. Then we have

$$
\left|v_{j}(t)\right|_{k-1} \leqslant\left|\Phi\left[u_{j}\right](t)\right|_{k} \leqslant R
$$

for $t \in\left[0, T_{2}\right] ; j=1,2$. Let $w:=v_{1}-v_{2}$. Since $v_{j}$ satisfies (1.15) with $u=u_{j}(j=1,2)$, it holds that

$$
\begin{array}{ll}
\partial_{t}^{2} w(t)+\mathscr{A}_{u_{1}}(t) w(t)+\mathscr{B}_{u_{1}}(t) \partial_{t} w(t) \\
=g_{u_{1}}(t)-g_{u_{2}}(t)+\left[\mathscr{A}_{u_{2}}(t)-\mathscr{A}_{u_{1}}(t)\right] v_{2}(t) \\
\quad+\left[\mathscr{B}_{u_{2}}(t)-\mathscr{B}_{u_{1}}(t)\right] \partial_{t} v_{2}(t) & \text { for } t \in\left[0, T_{2}\right], \\
w(t) \in \mathscr{H}^{m}(\Omega) & \text { for } t \in\left[0, T_{2}\right], \\
\partial_{t}^{j} w(t)=0 & \text { for } j=0, \ldots, k .
\end{array}
$$

We estimate the right-hand side of (4.15). From Lemma A.1 and Corollary A.3 we conclude that

$$
\begin{aligned}
& \left|g_{u_{1}}(t)-g_{u_{2}}(t)\right|_{k-3} \leqslant d_{1}\left|u_{1}(t)-u_{2}(t)\right|_{k-1} \leqslant d_{1}|| u_{1}-u_{2}|| \tilde{r}_{k-1, T_{2}}, \\
& \left|g_{u_{1}}(t)-g_{u_{2}}(t)\right|_{k-4} \leqslant d_{2}\left|u_{1}(t)-u_{2}(t)\right|_{k-2} \leqslant d_{2} T_{2}||\left|u_{1}-u_{2} \|\right|_{k-1}, T_{2},
\end{aligned}
$$

for $t \in\left[0, T_{2}\right]$, since

$$
\begin{aligned}
\left\|\partial_{i}^{j} u_{1}(t)-\partial_{t}^{j} u_{2}(t)\right\|_{(k-2-j) m} & \leqslant \int_{0}^{t}\left\|\partial_{t}^{j+1}\left[u_{1}(\tau)-u_{2}(\tau)\right]\right\|_{(k-2-j) m} \mathrm{~d} \tau \\
& \leqslant T_{2}\left\|u_{1}-u_{2}\right\| \|_{k-1, T_{2}}
\end{aligned}
$$

for $j=0, \ldots, k-2$. Here and in the following $d_{j}(j=1,2, \ldots)$ denote positive constants depending only on $R, k$ and $\left\|\partial_{t} f\right\|_{k-3, T}$.

Let $t \in\left[0, T_{2}\right]$. From Lemma A.1 and Corollary A.3 we obtain that

$$
\begin{aligned}
& \left|\left[a_{\alpha}\left(t . ., \mathscr{D} u_{2}(t)\right)-a_{\alpha}\left(t, ., \mathscr{D} u_{1}(t)\right)\right] \partial_{x}^{\alpha} v_{2}(t)\right|_{k-3}^{\tilde{k}} \\
& \leqslant a_{\alpha}\left(t, ., \mathscr{D} u_{2}(t)-\left.a_{\alpha}\left(t, ., \mathscr{D} u_{1}(t)\right)\right|_{k-3} ^{\tilde{k}}\left|v_{2}(t)\right|_{k-1}\right. \\
& \leqslant d_{4}\left|u_{1}(t)-u_{2}(t)\right|_{k-1}^{-}
\end{aligned}
$$


for $|\alpha| \leqslant 2 m$. This implies that

$$
\left|\left[\mathscr{A}_{u_{2}}(t)-\mathscr{A}_{u_{1}}(t)\right] v_{2}(t)\right|_{k-3}^{\tilde{z}} \leqslant d_{5}||\left|u_{1}-u_{2}\right| \mid \tilde{k}_{k-1, T_{2}} .
$$

In the same way it can be shown with (4.18) that

$$
\left|\left[\mathscr{A}_{u_{2}}(t)-\mathscr{A}_{u_{1}}(t)\right] v_{2}(t)\right|_{k-4} \leqslant d_{6} T_{2}\left|\left\|u_{1}-u_{2}|\||_{k-1, T_{2}}^{\tilde{s}}\right.\right. \text {. }
$$

Analogous estimates hold for $\left[\mathscr{B}_{u_{2}}(t)-\mathscr{B}_{u_{1}}(t)\right] v_{2}(t)$. Note that for $\varphi \in \tilde{\mathscr{C}}_{T_{2}}^{k-3}$

$$
\left\|\partial_{t}^{k-3} \varphi(t)\right\|+|\varphi(t)|_{k-4} \leqslant|\varphi(t)|_{k-3} \text { for } t \in\left[0, T_{2}\right] \text {. }
$$

We apply Theorem 1.1 of [6] to (4.15)-(4.17) and obtain

$$
\begin{aligned}
\left|v_{1}(t)-v_{2}(t)\right|_{k-2} & \leqslant d_{7} \exp \left(d_{8} t\right) \int_{0}^{t}\left\|u_{1}-u_{2} \mid\right\| \tilde{k}_{k-1, T_{2}} \mathrm{~d} \tau \\
& +d_{9} T_{2}\left\|\mid u_{1}-u_{2}\right\| \tilde{k}_{k-1}, T_{2} \\
& \leqslant\left(d_{7}+d_{9}\right) T_{2} \exp \left(d_{8} T_{2}\right)\left\|u_{1}-u_{2}\right\| \tilde{k}_{k-1, T_{2}} .
\end{aligned}
$$

Using (1.17) we conclude that

$$
\begin{aligned}
\left|\Phi\left[u_{1}\right](t)-\Phi\left[u_{2}\right](t)\right|_{k-1} & \leqslant \int_{0}^{t}\left\|v_{1}(\tau)-v_{2}(\tau)\right\|_{(k-2) m} \mathrm{~d} \tau+\left|v_{1}(t)-v_{2}(t)\right|_{k-2} \\
& \leqslant\left(d_{7}+d_{9}\right)\left(T_{2}^{2}+T_{2}\right) \exp \left(d_{8} T_{2}\right)\left\|u_{1}-u_{2}\right\| \|_{k-1, T_{2}}
\end{aligned}
$$

for $t \in\left[0, T_{2}\right]$. We choose $T_{2}>0$ so small that

$$
\left(d_{7}+d_{9}\right)\left(T_{2}^{2}+T_{2}\right) \exp \left(d_{8} T_{2}\right) \leqslant \frac{1}{2} .
$$

Then (4.19) implies (4.13) and Lemma 4.2 is proved.

By Lemma 4.1 and Lemma 4.2 we obtain the existence of a fixed point of $\Phi$ in the following way: suppose that all the assumptions of Lemma 4.1 are satisfied. Let $u_{0} \in \tilde{\mathscr{C}}_{T}^{k}$ be a function with $\partial_{i}^{j}(0)=u^{j}$ for $j=0, \ldots, k-1$ (compare, for example, Lemma A.8). We choose

$$
R:=\max \left\{\left\|u_{0}\right\| \tilde{k}_{k, T}, d_{12}+2 d_{11} \mathrm{e}\right\}
$$

where $d_{11}$ and $d_{12}$ are the constants of (4.11). Note that $R$ depends only on $k$ and $\tilde{N}_{k}$. Furthermore, we set

$$
T^{\prime}:=\min \left\{T_{1}, T_{2}\right\},
$$

with $T_{1}, T_{2}$ being the numbers of Lemma 4.1 and Lemma 4.2. Then the following statements hold:

(i) $\mathscr{M}\left(R, T^{\prime}\right) \neq \varnothing\left(\right.$ since $u_{0} \in \mathscr{M}\left(R, T^{\prime}\right)$.

(ii) $\Phi$ maps $\mathscr{M}\left(R, T^{\prime}\right)$ into itself.

(iii) For $u_{1}, u_{2} \in \mathscr{M}\left(R, T^{\prime}\right)$ (4.13) holds with $T_{2}$ being replaced by $T^{\prime}$.

The proof of Banach's fixed-point theorem yields the existence of a fixed-point $u \in \tilde{\mathscr{C}}_{T}^{k-1}$ of $\Phi$.

In order to prove the uniqueness of a fixed point of $\Phi$ in $\tilde{\mathscr{C}}_{T^{\prime}}^{k}$, we suppose that all the assumptions of Lemma 4.1 are satisfied. Let $u_{1}, u_{2} \in \tilde{\mathscr{C}}_{T}^{k}$ be two fixed points of $\Phi$. We 
choose

$$
R:=\max \left\{\left\|u_{1}\right\| \tilde{k}_{k}, T^{\prime},\left\|u_{2}\right\| \tilde{k}_{\boldsymbol{k}, T^{\prime}}\right\} .
$$

By $\Phi\left[u_{j}\right]=u_{j}(j=1,2)$ and Lemma 4.2 it follows that $u_{1}(t)=u_{2}(t)$ in some interval $\left[0, T_{2}\right]$. Note that $T_{2}>0$ depends only $R$. Hence a further application of Lemma 4.2 yields $u_{1}(t)=u_{2}(t)$ in $\left[0,2 T_{2}\right]$. After a finite number of steps we obtain $u_{1}(t)=u_{2}(t)$ in $\left[0, T^{\prime}\right]$. We have proved the following theorem.

Lemma 4.3. Let all the assumptions of Lemma 4.1 be satisfied. Then there exists a $T^{\prime}>0$ depending only on $k$ and $\tilde{N}_{k}$ such that $\Phi$ has a fixed point $u \in \tilde{\mathscr{C}}_{T}^{k-1}$. If $k \geqslant[n / 2 m]$ +6, then this fixed point is unique in $\tilde{\mathscr{C}}_{T}^{k-1}$.

\section{The fixed point of $\Phi$ and the solution}

Let $u \in \tilde{\mathscr{C}}_{T}^{k}$ with $k \geqslant[n / 2 m]+4$ be a fixed point of $\Phi$. Note that

$$
\bar{D}_{x}^{j m} u \in C_{b}^{2-j}([0, T] \times \bar{\Omega}) \quad j=0,1,2
$$

by the following lemma:

Lemma 5.1. Let $k \geqslant k_{2}:=[n / 2 m]+1$ and let $u \in \mathscr{C}_{T}^{k}$. Then it holds that

$$
\bar{D}_{x}^{j m} u \in C_{b}^{k-k_{2}-j}([0, T] \times \bar{\Omega}) \quad j=0, \ldots, k-k_{2} .
$$

We shall give the proof of Lemma 5.1 at the end of this section. Consider the fixed point $u \in \mathscr{C}_{T}^{k}$ of $\Phi$. It follows from the definition of $\Phi$ that $(u, v)=\left(u, \partial_{t} u\right)$ is a solution of (1.15) and (1.16). By the definition of $\mathscr{A}_{u}, \mathscr{B}_{u}$ and $g_{u}$ (compare (2.7)-(2.11)) we obtain from $v=\partial_{t} u$ and (1.15) that

$$
\begin{aligned}
0= & \int_{0}^{t}\left[\partial_{t}^{3} u(\tau, x)+\mathscr{A}_{u}(\tau) \partial_{t} u(\tau, x)+\mathscr{B}_{u}(\tau) \partial_{t}^{2} u(\tau, x)\right. \\
& \left.-g_{u}(\tau, x)\right] \frac{\partial F}{\partial \mu_{2}}(\tau, x, \mathscr{D} u(\tau, x)) \mathrm{d} \tau \\
= & \int_{0}^{t}\left(\frac{\partial}{\partial \tau}[F(\tau, x, \mathscr{D} u(\tau, x))-f(\tau, x)]\right) \mathrm{d} \tau \\
= & F(t, x, \mathscr{D} u(t, x))-f(t, x)-F(0, x, \mathscr{D} u(0, x))+f(0, x)
\end{aligned}
$$

for $(t, x) \in[0, T] \times \Omega$. Taking into account the fact that $F(0, x, \mathscr{D} u(0, x))=f(0, x)$ by (1.10), we conclude that (1.1) holds

Note that $\partial_{t} u(t)=v(t) \in \mathscr{H}^{m}(\Omega) \cap C_{b}^{m}(\bar{\Omega})$ for $t \in[0, T]$. Therefore, the following relation holds:

$$
\bar{D}_{x}^{m-1} v(t, x)=0 \text { on }[0, T] \times \partial \Omega .
$$

Since the same holds for $u^{0}(x)$, we obtain from

$$
u(t, x)=u^{0}(x)+\int_{0}^{t} v(\tau, x) \mathrm{d} \tau
$$

that $u$ satisfies (1.2). Furthermore, (1.3) holds. Hence $u$ is a classical solution of (1.1)-(1.3). In addition to this it follows from Lemma A.7 that $u \in \mathscr{C}_{T}^{k}$. 
On the other hand, every solution $u \in \mathscr{C}_{T}^{k}$ of (1.1)-(1.3) is a fixed point of $\Phi$, which follows from the construction of problem (1.15) and (1.16). Together with Lemma 4.3 we have proved the following lemma.

Lemma 5.2. Let all assumptions of 4.1 be satisfied. Then there exists a $T^{\prime}>0$ depending only on $k$ and $\tilde{N}_{k}$ such that (1.1)-(1.3) has a classical solution, $u \in \mathscr{C}_{T^{\prime}}^{k-1}$. Furthermore, it holds

$$
\bar{D}_{x}^{j m} u \in C_{b}^{k-k_{2}-1-j}\left(\left[0, T^{\prime}\right] \times \bar{\Omega}\right) \text { for } j=0, \ldots, k-k_{2}-1
$$

$\left(k_{2}=[n / 2 m]+1\right)$. If $k \geqslant[n / 2 m]+6$, then $u$ is unique in $\mathscr{C}_{T}^{k-1}$.

Remark. Lemma 5.2 differs from Theorem 1.1 in two points. First, if all the assumptions of Theorem 1.1 are satisfied, then Lemma 5.2 gives a solution $u \in \mathscr{C}_{T}^{k-1}$ instead of $u \in \mathscr{C}_{T}^{k}$. The second difference is that $T^{\prime}$ in Lemma 5.2 depends on $k$. Both problems will be solved in the next section.

Proof of Lemma 5.1. We prove that for $v \in C\left([0, T], H^{k_{2} m}(\Omega)\right)$ that the following holds:

$$
v \in C_{b}([0, T] \times \overline{\mathbf{\Omega}}) .
$$

Note that $k_{2} m \geqslant[n / 2]+1$. Hence Sobolev's lemma yields

$$
v(t) \in H^{k_{2} m}(\Omega) \subset C_{b}(\bar{\Omega}) \text { for } t \in[0, T] .
$$

Moreover, we obtain that

$$
\left|v\left(t_{1}, x\right)-v\left(t_{2}, x\right)\right| \leqslant c\left\|v\left(t_{1}\right)-v\left(t_{2}\right)\right\|_{k_{2} m} \rightarrow 0 \text { as } t_{1} \rightarrow t_{2}
$$

for $x \in \bar{\Omega}$. Since (5.6) is uniform with respect to $x \in \bar{\Omega}$, we conclude from (5.5) and (5.6) that (5.4) holds.

Let $k \geqslant k_{2}, u \in \mathscr{C}_{T}^{k}$. Then it holds that $\partial_{x}^{\alpha} \bar{D}_{x}^{j m} \partial_{t}^{i} u \in C\left([0, T], H^{k_{2} m}(\Omega)\right)$ for $|\alpha|+i \leqslant k$ $-k_{2}-j$. Thus we obtain by (5.4) that (5.2) is valid.

\section{Higher regularity and the proof of Theorem 1.1}

The gap between Lemma 5.2 and Theorem 1.1 will be bridged by the following lemma.

Lemma 6.1. Let $k \geqslant k_{0}=[n / 2 m]+6$ and let all the assumptions of Lemma 4.1 be satisfied. If $u \in \mathscr{C}_{T}^{k-1}$ is a solution of (1.1)-(1.3), then $u \in \mathscr{C}_{T}^{k}$.

Before proving Lemma 6.1 we consider Theorem 1.1. Let $k \geqslant k_{0}$ and let all the assumptions of Theorem 1.1 be satisfied. Lemma 5.2 gives us the existence of a $T^{\prime}>0$ such that (1.1)-(1.3) has a solution $u \in \mathscr{C}_{T}^{k_{0}}-1$. This $T^{\prime}$ depends only on $\tilde{N}_{k_{0}}$. Note that $\tilde{N}_{k_{0}} \leqslant c N$ by Lemma 3.3. Hence $T^{\prime}$ depends only on $N$. From Lemma 6.1 we obtain that $u \in \mathscr{C}_{T^{\prime}}^{k_{0}}$. Further application of Lemma 6.1 yields successively $u \in \mathscr{C}_{T}^{k_{0}+1}, \ldots, \mathscr{C}_{T}^{k}$. Finally it follows from Lemma 5.1 that $u$ has the regularity property (5.2), so that (1.13) follows if the assumptions of Theorem 1.1 are satisfied for every $k \geqslant k_{0}$. This proves Theorem 1.1 .

Proof of Lemma 6.1. This proof is a modification of a proof contained in [7]. Let $u \in \mathscr{C}_{T}^{k-1}$ be a solution of (1.1)-(1.3). Then (1.15) and (1.16) hold with $v=\partial_{t} u$. We 
differentiate (1.15) twice with respect to $t$ :

$$
\begin{aligned}
& \partial_{t}^{5} u(t)+\mathscr{A}_{u}(t) \partial_{t}^{3} u(t)+\mathscr{B}_{u}(t) \partial_{t}^{4} u(t) \\
& =\partial_{t}^{2} g_{u}(t)-2\left[\partial_{t} \mathscr{A}_{u}(t)\right] \partial_{t}^{2} u(t)-2\left[\partial_{t} \mathscr{B}_{u}(t)\right] \partial_{t}^{3} u(t) \\
& \quad-\left[\partial_{t}^{2} \mathscr{A}_{u}(t)\right] \partial_{t} u(t)-\left[\partial_{t}^{2} \mathscr{B}_{u}(t)\right] \partial_{t}^{2} u(t) \text { for } t \in[0, T] .
\end{aligned}
$$

It holds that (compare (2.11))

$$
\begin{aligned}
\partial_{t}^{2} g_{u}(t)= & \partial_{t}^{2}[g(t, ., \mathscr{D} u(t))]=\nabla_{\mu} g(t, ., \mathscr{D} u(t)) \cdot \mathscr{D} \partial_{t}^{2} u(t)+\tilde{g}(t) \\
= & \nabla_{\mu_{0}} g(t, ., \mathscr{D} u(t)) \cdot \bar{D}_{x}^{2 m} \partial_{t}^{2} u(t)+\nabla_{\mu_{1}} g(t, ., \mathscr{D} u(t)) \cdot \bar{D}_{x}^{m} \partial_{1}^{3} u(t) \\
& +\frac{\partial g}{\partial \mu_{2}}(t, ., \mathscr{D} u(t)) \partial_{t}^{4} u(t)+\tilde{g}(t),
\end{aligned}
$$

where $\nabla_{\mu} g:=\left(\partial g / \partial \mu_{0}^{(\alpha)}, \partial g / \partial \mu_{1}^{(\beta)}, \partial g / \partial \mu_{2}:|\alpha| \leqslant 2 m,|\beta| \leqslant m\right)$ and

$$
\begin{aligned}
\tilde{g}(t):= & \left(\partial_{t}^{2} g\right)(t, ., \mathscr{D} u(t))+2 \nabla_{\mu}\left(\partial_{t} g\right)(t, ., \mathscr{D} u(t)) \cdot \mathscr{D} \partial_{t} u(t) \\
& +\sum_{|\gamma|=2} \nabla_{\mu}^{\gamma} g(t, ., \mathscr{D} u(t)) \cdot\left(\mathscr{D} \partial_{t} u(t)\right)^{\gamma} .
\end{aligned}
$$

In the same way we obtain

$$
\begin{aligned}
& \partial_{t}^{2} \mathscr{A}_{u}(t)=\sum_{|\alpha| \leqslant 2 m}\left[\nabla_{\mu} a_{x}(t, ., \mathscr{D} u(t)) \cdot \mathscr{D} \partial_{t}^{2} u(t)+\tilde{a}_{\alpha}(t)\right] \partial_{x}^{\alpha} \\
& \partial_{t}^{2} \mathscr{B}_{u}(t)=\sum_{|\beta| \leqslant m}\left[\nabla_{\mu} b_{\beta}(t, \ldots \mathscr{D} u(t)) \cdot \mathscr{D} \partial_{t}^{2} u(t)+\tilde{b}_{\beta}(t)\right] \partial_{x}^{\beta}
\end{aligned}
$$

with $\tilde{a}_{\alpha}$ and $\tilde{b}_{\beta}$ being defined analogously to $\tilde{g}$. We set $w(t):=\partial_{t}^{2} u(t)$ and rewrite (6.1) as

$$
\begin{aligned}
& \partial_{t}^{3} w(t)+\mathscr{A}^{\prime}(t) \partial_{t} w(t)+\mathscr{B}^{\prime}(t) \partial_{t}^{2} w(t) \\
& =h(t)-\mathscr{G}(t) w(t)-2\left[\partial_{t} \mathscr{B}_{u}(t)\right] \partial_{t} w(t)
\end{aligned}
$$

with

$$
\begin{aligned}
\mathscr{A}^{\prime}(t)= & \sum_{|\gamma| \leqslant 2 m} a_{\gamma}^{\prime}(t) \partial_{x}^{\gamma} \\
:= & \mathscr{A}_{u}(t)-\nabla_{\mu_{1}} g(t, ., \mathscr{D} u(t)) \cdot \bar{D}_{x}^{m} \\
& +\left(\sum_{|\alpha| \leqslant 2 m}\left[\partial_{x}^{\alpha} \partial_{t} u(t)\right] \nabla_{\mu_{1}} a_{\alpha}((t, ., \mathscr{D} u(t))) \cdot \bar{D}_{x}^{m}\right. \\
& +\left(\sum_{|\beta| \leqslant m}\left[\partial_{x}^{\beta} \partial_{t}^{2} u(t)\right] \nabla_{\mu_{1}} b_{\beta}((t, ., \mathscr{D} u(t))) \cdot \bar{D}_{x}^{m},\right. \\
\mathscr{B}^{\prime}(t)= & \sum_{|\delta| \leqslant m} b_{\delta}^{\prime}(t) \partial_{x}^{\delta} \\
:= & \mathscr{B}{ }_{u}(t)-\frac{\partial g}{\partial \mu_{2}}(t, ., \mathscr{D} u(t))+\sum_{|z| \leqslant 2 m}\left[\partial_{x}^{\alpha} \partial_{t} u(t)\right] \frac{\partial a_{\alpha}}{\partial \mu_{2}}(t, ., \mathscr{D} u(t)) \\
& +\sum_{|\beta| \leqslant m}\left[\partial_{x}^{\beta} \partial_{t}^{2} u(t)\right] \frac{\partial b_{\beta}}{\partial \mu_{2}}(t, ., \mathscr{D} u(t)), \\
h(t):= & \tilde{g}(t)-\sum_{|\alpha| \leqslant 2 m} \tilde{a}_{\alpha}(t) \partial_{x}^{\alpha} \partial_{t} u(t)-\sum_{|\beta| \leqslant m} \tilde{b}_{\beta}(t) \partial_{x}^{\beta} \partial_{t}^{2} u(t)
\end{aligned}
$$


and

$$
\begin{aligned}
\mathscr{G}(t)= & \sum_{|\gamma| \leqslant 2 m} g_{y}(t) \partial_{x}^{\gamma} \\
:= & 2\left[\partial_{,} \mathscr{A}_{u}(t)\right]-\nabla_{\mu_{0}} g(t, ., \mathscr{D} u(t)) \cdot \bar{D}_{x}^{2 m} \\
& +\left(\sum_{|\alpha| \leqslant 2 m}\left[\partial_{x}^{\alpha} \partial_{t} u(t)\right] \nabla_{\mu_{0}} a_{\alpha}(t, ., \mathscr{D} u(t))\right) \cdot \bar{D}_{x}^{2 m} \\
& +\left(\sum_{|\beta| \leqslant m}\left[\partial_{x}^{\beta} \partial_{t}^{2} u(t)\right] \nabla_{\mu_{0}} b_{\beta}(t, ., \mathscr{D} u(t))\right) \cdot \bar{D}_{x}^{2 m}
\end{aligned}
$$

Note that $a_{\alpha}^{\prime}(t)=a_{\alpha}(t, ., \mathscr{D} u(t))$ for $|\alpha|=2 m$, so that $\mathscr{A}^{\prime}(t)$ is a family of uniformly elliptic operators. Furthermore, $b_{\beta}^{\prime}(t)=b_{\beta}(t, ., \mathscr{D} u(t))$ for $|\beta| \geqslant 1$. Hence (2.14) holds with $\mathscr{B}_{u}(t)$ being replaced by $\mathscr{B}^{\prime}(t)$. From Lemma A.1 and Corollary A.3 we obtain that $h \in \mathscr{C}_{T}^{k-4}$ and that the coefficients $a_{a}^{\prime}(t)$ have a representation

$$
a_{\alpha}^{\prime}(t, x)=p_{\alpha}(t, x)+q_{\alpha}(t, x)
$$

$((t, x) \in[0, T] \times \Omega,|\alpha| \leqslant 2 m)$ such that

$$
\left.\begin{array}{l}
p_{\alpha} \in C_{b}^{(k-2) m}([0, T] \times \bar{\Omega}), \\
q_{\alpha} \in \mathscr{C}_{T}^{k-4} .
\end{array}\right\}
$$

The same holds for $b_{\beta}^{\prime}(t), g_{\gamma}(t), \partial_{t} b_{\beta}(t, ., \mathscr{D} u(t))$.

From (1.15) and (6.4) we conclude that $w=\partial_{t}^{2} u \in \mathscr{C}_{T}^{k-3}$ is a solution of the following linear problem

$$
\begin{array}{ll}
\partial_{t}^{3} w(t)+\mathscr{A}^{\prime}(t) \partial_{t} w(t)+\mathscr{B}^{\prime}(t) \partial_{t}^{2} w(t) & \\
=h(t)-\mathscr{G}(t) w(t)-2\left[\partial_{t} \mathscr{B}_{u}(t)\right] \partial_{t} w(t) & \text { for } t \in[0, T], \\
w(t) \in \stackrel{\circ}{ }^{m}(\Omega) & \text { for } t \in[0, T], \\
w(0)=u^{2}, \quad \partial_{t} w(0)=u^{3}, \quad \partial_{t}^{2} w(0)=u^{4} . &
\end{array}
$$

We prove the existence of a solution $w \in \tilde{\mathscr{C}}_{T}^{k-2}$ of $(6.11)$ by the method of successive approximation (compare [3]). Let $w_{0} \in \tilde{\mathscr{C}}_{T}^{k-2}$ with $\partial_{t}^{j} w_{0}(0)=u^{j+2}$ for $j=0, \ldots$, $k-3$ (compare Lemma A.8). We define $w_{j}$ for $j \geqslant 1$ successively by

$$
\begin{array}{ll}
\partial_{i}^{2} v_{j+1}(t)+\mathscr{A}^{\prime}(t) v_{j+1}(t)+\mathscr{B}(t) \partial_{t} v_{j+1}(t) & \\
=h(t)-\mathscr{G}(t) w_{j}(t)-2\left[\partial_{t} \mathscr{B}_{u}(t)\right] \partial_{t} w_{j}(t) & \text { for } t \in[0, T], \\
v_{j+1}(t) \in \dot{H}^{m}(\Omega) & \text { for } t \in[0, T], \\
v_{j+1}(0)=u^{3}, \quad \partial_{t} v_{j+1}(0)=u^{4}, & \\
w_{j+1}(t):=u^{2}+\int_{0}^{t} v_{j+1}(\tau) \mathrm{d} \tau & \text { for } t \in[0, T] .
\end{array}
$$

We prove $w_{j} \in \tilde{\mathscr{C}}_{T}^{k-2}$ by induction. Let $w_{j} \in \tilde{\mathscr{C}}_{T}^{k-2}$, then

$$
h-\mathscr{G}(\cdot) w_{j}-2\left[\partial_{t} \mathscr{B}_{u}(\cdot)\right] \partial_{t} w_{j} \in \tilde{\mathscr{C}}_{T}^{k-4} \text {. }
$$

We apply Theorem 1.1 of [6] to problem (6.12) and obtain $v_{j+1} \in \mathscr{C}_{T}^{k-3}$. Hence (6.13) implies $w_{j+1} \in \widetilde{\mathscr{C}}_{T}^{k-2}$.

Note that $\partial_{i}^{v} w_{j}(0)=u^{v+2}(v=0, \ldots, k-2)$ for every $j \in \mathbb{N}$. We consider the 
differences $v_{j+1}-v_{j}, w_{j+1}-w_{j}$. The following relations hold

$$
\begin{array}{ll}
{\left[\partial_{t}^{2}+\mathscr{A}^{\prime}(t)+\mathscr{B}^{\prime}(t) \partial_{t}\right]\left[v_{j+1}(t)-v_{j}(t)\right]} & \\
=\left\{-\mathscr{G}(t)-2\left[\partial_{t} \mathscr{B}_{u}(t)\right] \partial_{t}\right\}\left[w_{j}(t)-w_{j-1}(t)\right] & \text { for } t \in[0, T], \\
v_{j+1}(t)-v_{j}(t) \in \dot{H}^{m}(\Omega) & \text { for } t \in[0, T], \\
\partial_{t}^{v} v_{j+1}(0)-\partial_{t}^{v} v_{j}(0)=0 & \text { for } v=0, \ldots, k-2
\end{array}
$$

and

$$
w_{j+1}(t)-w_{j}(t)=\int_{0}^{t}\left[v_{j+1}(\tau)-v_{j}(\tau)\right] \mathrm{d} \tau \text { for } t \in[0, T] .
$$

With Corollary A.3 we conclude that

$$
\begin{aligned}
\left\{\left.\left\{\mathscr{G}(t)+2\left[\partial_{t} \mathscr{B}_{u}(t)\right] \partial_{i}\right\}\left(w_{j}(t)-w_{j-1}(t)\right)\right|_{k-4}\right. & \leqslant c_{1}\left|w_{j}(t)-w_{j-1}(t)\right|_{k-2}, \\
\left|\left\{\mathscr{G}(t)+2\left[\partial_{t} \mathscr{B}_{u}(t)\right] \partial_{t}\right\}\left(w_{j}(t)-w_{j-1}(t)\right)\right|_{k-5} & \leqslant c_{2}\left|w_{j}(t)-w_{j-1}(t)\right|_{k-3} \\
& \leqslant c_{2} \int_{0}^{t} \mid w_{j}(\tau)-w_{j-1}(\tau) \tilde{r}_{k-2} \mathrm{~d} \tau .
\end{aligned}
$$

Applying the energy estimate of Theorem 1.1 in [6] to (6.14) we obtain that

$$
\left|v_{j+1}(t)-v_{j}(t)\right|_{k-3} \leqslant c_{3} \int_{0}^{t}\left|w_{j}(\tau)-w_{j-1}(\tau)\right|_{k-2} \mathrm{~d} \tau
$$

with $c_{3}>0$ depending on $T$. With (6.15) it follows that

$$
\left|w_{j+1}(t)-w_{j}(t)\right|_{k-2} \leqslant c_{4} \int_{0}^{t}\left|w_{j}(\tau)-w_{j-1}(\tau)\right|_{k-2}^{\tau} \mathrm{d} \tau
$$

with $c_{4}:=c_{3} T$. By induction we obtain from (6.16) that

$$
\left|w_{j+1}(t)-w_{j}(t)\right|_{k-2} \leqslant \frac{\left(c_{4} t\right)^{j}}{j !}\left\|\mid w_{1}-w_{0}\right\| \tilde{r}_{k-2, T}
$$

for $t \in[0, T]$. This implies that $\left\{w_{j}\right\}$ converges in $\mathscr{C}_{T}^{k-2}$. We denote the limit by $w$. Since $v_{j}=\partial_{t} w_{j}$, the sequence $\left\{v_{j}\right\}$ converges in $\mathscr{C}_{T}^{k-3}$ to $\partial_{t} w$. Hence it follows from (6.12) and (6.13) that $w \in \tilde{\mathscr{C}}_{T}^{k-2}$ is a solution of (6.11).

It remains to be shown that $w=\partial_{t}^{2} u$. Let $w^{(1)}, w^{(2)} \in \mathscr{C}_{T}^{k-3}$ be two solutions of (6.11). Then (6.14) and (6.15) hold with $w_{j}-w_{j-1}, v_{j+1}-v_{j}$ and $w_{j+1}-w_{j}$ being replaced by $w^{(1)}-w^{(2)}, \partial_{t} w^{(1)}-\partial_{t} w^{(2)}$ and $w^{(1)}-w^{(2)}$, respectively. The same argument that leads to (6.17) gives us

$$
\left|w^{(1)}(t)-w^{(2)}(t)\right|_{k-3} \leqslant\left(\frac{c_{4} t}{j !}\right)^{j}\left\|w^{(2)}-w^{(1)} \mid\right\|_{k-3, T}^{\sim} \text { for } t \in[0, T]
$$

for every $j \in \mathbb{N}$. It follows that $w^{(1)}=w^{(2)}$. This proves $\partial_{\imath}^{2} u=w \in \tilde{\mathscr{C}}_{T}^{k-2}$.

We rewrite (1.15) as

$$
\mathscr{A}_{u}(t) \partial_{t} u(t)=g_{u}(t)-\partial_{t}^{3} u(t)-\mathscr{B}_{u}(t) \partial_{t}^{2} u(t)
$$

Differentiation with respect to $t$ yields

$$
\begin{aligned}
\mathscr{A}_{u}(t) \partial_{t}^{2} u(t)= & \partial_{t} g_{u}(t)-\partial_{t}^{4} u(t)-\mathscr{B}_{u}(t) \partial_{t}^{3} u(t) \\
& -\left[\partial_{t} \mathscr{A}_{u}(t)\right] \partial_{t} u(t)-\left[\mathscr{B}_{u}(t)\right] \partial_{t}^{2} u(t) .
\end{aligned}
$$


Taking into account that $u \in \mathscr{C}_{T}^{k-1}, \partial_{t}^{2} u \in \tilde{\mathscr{C}}_{T}^{k-2}$ we conclude that the right-hand side of (6.19) is an element of $C\left([0, T], H^{(k-4) m}(\Omega)\right)$. The elliptic regularity theory (compare [6]) yields $\partial_{t}^{2} u \in C\left([0, T], H^{(k-2) m}(\Omega)\right)$. Hence we have $\partial_{t}^{2} u \in \mathscr{C}_{T}^{k-2}$.

In the same way we conclude from (6.18) that $\partial_{t} u \in \mathscr{C}_{T}^{k-1}$ and therefore $u \in \tilde{\mathscr{C}}_{T}^{k}$. Finally, it follows by Lemma A.7 that $u \in \mathscr{C}_{T}^{k}$.

\section{The existence interval for small data}

In this section we study solutions $u \in \mathscr{C}_{T}^{k}\left(k \geqslant k_{0}\right)$ of $(1.1)-(1.3)$ with $\|u\|_{\boldsymbol{k}_{0}, T} \leqslant R_{0}$, where $R_{0}>0$ is chosen so that

$$
|\mathscr{D} u(t, x)| \leqslant c\|\mathscr{D} u(t)\|_{\left(k_{0}-3\right) m} \leqslant c R_{0} \leqslant \frac{1}{2} M \quad \text { for }(t, x) \in[0, T] \times \bar{\Omega}
$$

by Sobolev's lemma. We suppose that all the assumptions of Theorem 1.1 are satisfied for every $T>0$ and that

$$
\begin{aligned}
\tilde{N}:= & \sum_{j=0}^{k_{0}}\left\|u^{j}\right\|_{\left(k_{0}-j\right) m}+\int_{0}^{\infty}\left(\left|\partial_{t} f(t)\right|_{k_{0}-3}+\left\|\partial_{t}^{k_{0}-1} f(t)\right\|\right) \mathrm{d} t \\
& +\sup _{[\mathbf{0}, \infty)}\left|\partial_{t} f(t)\right|_{k_{0}-3} \leqslant \frac{1}{2} R_{0} .
\end{aligned}
$$

According to Theorem 1.1 there exists a $T>0$ and a solution $u \in \mathscr{C}_{T}^{k}$ of (1.1)-(1.3). Since $|u(0)|_{k_{0}}^{\tau_{0}} \leqslant \tilde{N} \leqslant \frac{1}{2} R_{0}$ by (7.2), we can choose $T$ so small that $|u(t)|_{k_{0}} \leqslant R_{0}$ for $t \in[0, T]$. We use $\Phi[u]=u$ to prove an a priori estimate for $|u(t)|_{k_{0}}$.

By (4.9) and $v=\partial_{t} \Phi[u]$ we have

$$
\begin{aligned}
& \left|\partial_{t} \Phi[u](t)\right|_{k_{0}-1} \leqslant d_{1} \exp \left(d_{2} t\right)\left[\left|\partial_{t} \Phi[u](0)\right|_{k_{0}-1}\right. \\
& \left.\quad+\int_{0}^{t}\left(\left\|\partial_{t}^{k_{0}-2} g_{u}(\tau)\right\|+\left|g_{u}(\tau)\right|_{k_{0}-3}\right) \mathrm{d} \tau\right]+d_{3}\left|g_{u}(t)\right|_{k_{0}-3} \quad \text { for } t \in[0, T] .
\end{aligned}
$$

Here and in the following we denote by $d_{j}(j=1,2, \ldots)$ positive constants that can be chosen independently of all $u \in \mathscr{C}_{T}^{k}$ with $\|u\|_{k_{0}, T}^{\tilde{x}_{1}} \leqslant R_{0}$. From (2.11) and $\partial_{t} F(t, x, 0)$ $=0$ on $[0, T] \times \bar{\Omega}$ we conclude by Lemma A.1 and Corollary A.4 that

$$
\begin{aligned}
\left|g_{u}(t)\right|_{k_{0}-3} & \leqslant d_{4}\left(\left|\partial_{t} f(t)\right|_{k_{0}-3}+|u(t)|_{k_{0}-1}\right), \\
\left\|\partial_{t}^{k_{0}-2} g_{u}(t)\right\| & \leqslant d_{5}\left(\left|\partial_{t} f(t)\right|_{k_{0}-3}+\left\|\partial_{t}^{k_{0}-1} f(t)\right\|+|u(t)|_{k_{0}}^{\tau_{0}}\right)
\end{aligned}
$$

and therefore

$$
\begin{aligned}
\int_{0}^{t}\left(\left\|\partial_{t}^{k_{0}-2} g_{u}(\tau)\right\|+\left|g_{u}(\tau)\right|_{k_{0}-3}\right) \mathrm{d} \tau & \leqslant\left(d_{4}+d_{5}\right)\left(\tilde{N}+\int_{0}^{t}|u(\tau)|_{k_{0}} \mathrm{~d} \tau\right), \\
\left|g_{u}(t)\right|_{k_{0}-3} & \leqslant d_{4}\left(\tilde{N}+|u(0)|_{k_{0}-1}+\int_{0}^{t}|u(\tau)|_{k_{0}} \mathrm{~d} \tau\right) \\
& \leqslant d_{6} \tilde{N}+d_{4} \int_{0}^{t}|u(\tau)|_{k_{0}} \mathrm{~d} \tau
\end{aligned}
$$

for $t \in[0, T]$. With $\left|\partial_{t} \Phi[u](0)\right|_{k_{0}-1} \leqslant \tilde{N}$ we obtain from (7.3) that

$$
\left|\partial_{t} \Phi[u](t)\right|_{k_{0}-1} \leqslant \exp \left(d_{2} t\right)\left(d_{8} \tilde{N}+d_{9} \int_{0}^{t}|u(\tau)|_{k_{0}} \mathrm{~d} \tau\right) \text { for } t \in[0, T]
$$


where we can choose $d_{2} \geqslant 1$. It follows that

$$
\begin{aligned}
|\Phi[u](t)|_{k_{0}} & \leqslant\|\Phi[u](0)\|_{\left(k_{0}-1\right) m}+\int_{0}^{t}\left\|\partial_{t} \Phi[u](\tau)\right\|_{\left(k_{0}-1\right) m} \mathrm{~d} \tau+\left|\partial_{t} \Phi[u](t)\right|_{k_{0}-1} \\
& \leqslant \tilde{N}+\exp \left(d_{2} t\right)\left(d_{8} \tilde{N}+d_{9} \int_{0}^{t}|u(\tau)|_{k_{0}} \mathrm{~d} \tau\right)\left(1+\frac{1}{d_{2}}\right) \\
& \leqslant \exp \left(d_{2} t\right)\left(d_{10} \tilde{N}+d_{11} \int_{0}^{t}|u(\tau)|_{k_{0}} \mathrm{~d} \tau\right) \text { for } t \in[0, T]
\end{aligned}
$$

From this and $|u(t)|_{k_{0}} \leqslant R_{0}$ we conclude by induction that

$$
\mid \Phi^{j}[u](t){\tilde{k_{0}}}_{\hat{N}} \leqslant \tilde{N} d_{10} \sum_{v=0}^{j-1} \frac{1}{v !}\left(\frac{d_{11}}{d_{2}}\right)^{v} \exp \left[d_{2}(v+1) t\right]+t R_{0} \frac{d_{11}^{j} \exp \left(j d_{2} t\right)}{(j-1) ! d_{2}^{j-1}}
$$

$(t \in[0, T] ; j=0,1, \ldots)$. Note that $\Phi^{j}[u]=u$. Letting $j \rightarrow \infty$ we obtain

$$
\begin{aligned}
|u(t)|_{k_{0}} & \leqslant \tilde{N} d_{10} \exp \left(d_{2} t+\frac{d_{11}}{d_{2}} \exp \left(d_{2} t\right)\right) \\
& \leqslant d_{10} \exp \left[\left(d_{11}+1\right) \exp \left(d_{2} t\right)\right] \tilde{N}
\end{aligned}
$$

for $t \in[0, T]$. We set

$$
T_{3}:=\frac{1}{d_{2}} \log \left(\frac{1}{d_{11}+1} \log \frac{R_{0}}{\tilde{N}}\right)
$$

for sufficiently small $\tilde{N}$. Then it holds that $\mid u(t) \tilde{k}_{k_{0}} \leqslant R_{0}$ for $t \in\left[0, T_{3}\right]$.

We denote by $\left[0, T_{4}\right]$ the largest interval on which the solution $u \in \mathscr{C}_{T}^{k}$ of (1.1)-(1.3) exists. It holds that $T_{4}>T_{3}$. In fact, if we assume $T_{4} \leqslant T_{3}$, then it follows with $\left|u\left(T_{4}\right)\right|_{k_{0}} \leqslant R_{0}$ and (7.1) that all the assumptions of Theorem 1.1 are satisfied in $\left[T_{4}, \infty\right)$. Hence there exists a $\Delta T>0$ and a solution

$$
\tilde{u} \in \bigcap_{j=0}^{k} C^{j}\left(\left[T_{4}, T_{4}+\Delta T\right], H^{(k-j) m}(\Omega)\right)
$$

Note that $\partial_{z}^{j} \tilde{u}\left(T_{4}\right)=\partial_{t}^{j} u\left(T_{4}\right)$ for $j=0, \ldots, k$ by the considerations of section 3 . Therefore we can extend the solution $u \in \mathscr{C}_{T_{4}}^{k}$ to a solution $u \in \mathscr{C}_{T_{4}+\Delta T}^{k}$ in contradiction to the definition of $T_{4}$. Thus we have proved $T_{4}>T_{3}=\mathcal{O}(\log \log (1 / \tilde{N}))$ as $\tilde{N} \downarrow 0$. Using Lemma 3.3 we obtain (1.14).

\section{Appendix}

In the first part of this section we study $G(t, x, \mathscr{D} u(t, x))$, where

$$
G \in C_{b}^{(k-2) m+1}\left([0, T] \times \bar{\Omega} \times\left\{\mu \in \mathbb{R}^{q(n, m)}\right\}\right)
$$

and $u \in \mathscr{C}_{T}^{k}$ for some $k \geqslant k_{2}:=[n / 2 m]+4$.

Lemma A.1. Let $k \geqslant k_{2}=[n / 2 m]+4$ and $G$ satisfying $(A .1)$ be given.

(i) We set $\tilde{G}(t, x):=G(t, x, \mathscr{D} u(t, x))-G(t, x, 0)$. If $u \in \tilde{\mathscr{C}}_{T}^{k} \cap \mathscr{C}_{T}^{k_{2}}$, then $\tilde{G} \in \tilde{\mathscr{C}}_{T}^{k-2}$ 
and

$$
\begin{aligned}
& |\tilde{G}(t)|_{k-2} \leqslant c_{1}\left(1+|u(t)|_{k-1}\right)^{(k-2) m}|u(t)|_{k}, \\
& |\tilde{G}(t)|_{k-3} \leqslant c_{2}\left(1+|u(t)|_{k-1}\right)^{(k-3) m}|u(t)|_{k-1}
\end{aligned}
$$

for $t \in[0, T]$ with $c_{1}, c_{2}>0$ depending only on $k, G$ and $\Omega$.

(ii) If $u_{1}, u_{2} \in \tilde{\mathscr{C}}_{T}^{k} \cap \mathscr{C}_{T}^{k_{2}}$, then

$$
\begin{aligned}
& \left|G\left(t, ., \mathscr{D} u_{1}(t)\right)-G\left(t, ., \mathscr{D} u_{2}(t)\right)\right|_{k-2} \leqslant c_{3}\left|u_{1}(t)-u_{2}(t)\right|_{k}, \\
& \left|G\left(t, ., \mathscr{D} u_{1}(t)\right)-G\left(t, ., \mathscr{D} u_{2}(t)\right)\right|_{k-3} \leqslant c_{4}\left|u_{1}(t)-u_{2}(t)\right|_{k-1},
\end{aligned}
$$

for $t \in[0, T]$, where $c_{3}, c_{4}>0$ depend only on $k, G, \Omega$ and $\left\|u_{1}\right\|\left\|_{k, T},\right\|\left\|u_{2}\right\| \tilde{k}_{k, T}$.

Before we can prove Lemma A.1 we need two lemmata given in the following.

Lemma A.2. Suppose that $i, j \in \mathbb{N}_{0}$ such that $r:=\min \{i, j, i+j-[n / 2]-1\} \geqslant 0$. Then for $f \in C\left([0, T], H^{i}(\Omega)\right), g \in C\left([0, T], H^{j}(\Omega)\right)$ it holds that $f g \in C\left([0, T], H^{r}(\Omega)\right)$ and

$$
\|f(t) g(t)\|_{r} \leqslant c\|f(t)\|_{i}\|g(t)\|_{j} \text { for } t \in[0, T]
$$

with $c>0$ depending only on $\Omega, i$ and $j$; here fg denotes the pointwise multiplication of $f$ and $g$.

Proof. Let $t \in[0, T]$ be fixed and suppose that $r=0$. We prove that

$$
\|f(t) g(t)\| \leqslant c\|f(t)\|_{i}\|g(t)\|_{j} \text { for } t \in[0, T] .
$$

If $i=[n / 2]+1$, then $f(t) \in C_{b}(\bar{\Omega})$ and $|f(t, x)| \leqslant c\|f(t)\|_{i}$ for $(t, x) \in[0, T] \times \bar{\Omega}$ by the lemma of Sobolev. Hence (A.7) follows immediately. The same holds if $j=[n / 2]+1$.

We suppose that $i, j \leqslant[n / 2]$ and $i+j=[n / 2]+1$. Note that $i, j \geqslant 1$. We set

$$
q:=\frac{2 n}{n-2 i}, \quad p:=\frac{n}{i}
$$

Then we have $2 / q+2 / p=1, q \geqslant 2$ and

$$
2 \leqslant p=\frac{n}{[n / 2]+1-j} \leqslant \frac{2 n}{n-2 j} .
$$

From Sobolev's lemma (compare [1]) it follows that $f(t) \in L_{q}(\Omega), g(t) \in L_{p}(\Omega)$ and

$$
\|f(t)\|_{L_{q}(\Omega)} \leqslant c\|f(t)\|_{i}, \quad\|g(t)\|_{L_{p}(\mathbf{\Omega})} \leqslant c\|g(t)\|_{j}
$$

for $t \in[0, T]$. Hölders inequality yields (A.7).

From (A.7) we conclude that

$$
\begin{aligned}
\left\|f\left(t_{1}\right) g\left(t_{1}\right)-f\left(t_{2}\right) g\left(t_{2}\right)\right\| \leqslant & \left\|f\left(t_{1}\right)\right\|_{i}\left\|g\left(t_{1}\right)-g\left(t_{2}\right)\right\|_{j} \\
& +\left\|f\left(t_{1}\right)-f\left(t_{2}\right)\right\|_{i}\left\|g\left(t_{2}\right)\right\|_{j}
\end{aligned}
$$

for $t_{1}, t_{2} \in[0, T]$ and hence $f g \in C\left([0, T], L_{2}(\Omega)\right)$. This proves Lemma A.2 in the case $r=0$. For $r>0$, Lemma A.2 follows by induction.

Corollary A.3 (i) Let $u \in \mathscr{C}_{T}^{k}, v \in \mathscr{C}_{T}^{l}$ with $r:=\min [\mathrm{k}, 1, \mathrm{k}+l-[n / 2 m]-1\} \geqslant 0$. Then $u v \in \mathscr{C}_{T}^{r}$ and

$$
|u(t) v(t)|_{r} \leqslant c|u(t)|_{k}|v(t)|_{l} \text { for } t \in[0, T]
$$


where $c>0$ depends only on $\Omega, k, l$ and $r$.

(ii) Let $u \in \tilde{\mathscr{C}}_{T}^{k}, v \in \tilde{\mathscr{C}}_{T}^{l}$ with $r:=\min \{k, l, k+l-[n / 2 m]-2\} \geqslant 1$. Then $u v \in \tilde{\mathscr{C}}_{T}^{r}$ and

$$
|u(t) v(t)|_{r}^{\tilde{s}} \leqslant c|u(t)|_{\dot{k}}|v(t)|_{i} \text { for } t \in[0, T],
$$

with $c>0$ depending on $\Omega, k, l$ and $r$.

Proof. Note that $(k+l) m \geqslant[n / 2]+1$ in case (i) and $(k+l-2) m \geqslant[n / 2]+1$ in case (ii), respectively. Corollary A.3 follows from Lemma A.2 by the Leibnitz rule and the definition of $\mathscr{C}_{T}^{k}$ and $\tilde{\mathscr{C}}_{T}^{k}$ (compare (1.7), (1.18), (2.3) and (2.5)).

Proof of Lemma A.1. At first we prove by induction that

$$
\left.\begin{array}{l}
\tilde{G} \in C\left([0, T], H^{l}(\Omega)\right), \\
\|\tilde{G}(t)\|_{l} \leqslant c_{1}\left(1+|u(t)|_{k-1}\right)^{l}|u(t)|_{k-1} \quad \text { for } t \in[0, T]
\end{array}\right\}
$$

for $l=0, \ldots,(k-3) m$.

It holds that

since

$$
\|\tilde{G}(t)\| \leqslant c_{2}|u(t)|_{2} \quad \text { for } t \in[0, T],
$$

$$
|G(t, x, \mu)-G(t, x, 0)| \leqslant c_{2}|\mu| \text { for }(t, x, \mu) \in[0, T] \times \bar{\Omega} \times \mathbb{R}^{q(n, m)} .
$$

In the same way we obtain from

$$
\begin{aligned}
\partial_{t} \tilde{G}(t, x)= & \left(\partial_{t} G\right)(t, x, \mathscr{D} u(t, x))-\left(\partial_{t} G\right)(t, x, 0) \\
& +\nabla_{\mu} G(t, x, \mathscr{D} u(t, x)) \cdot \mathscr{D} \partial_{t} u(t, x)
\end{aligned}
$$

that $\left\|\partial_{1} \tilde{G}(t)\right\| \leqslant c_{3}|u(t)|_{3}$ for $t \in[0, T]$. This implies $\tilde{G} \in C\left([0, T], L_{2}(\Omega)\right)=\mathscr{C}_{T}^{0}$ and (A.10) is proved for $l=0$. In the case $l=1$ we obtain from

$$
\begin{aligned}
\frac{\partial \tilde{G}(t, x)}{\partial x_{i}}= & \frac{\partial G}{\partial x_{i}}(t, x, \mathscr{D} u(t, x))-\frac{\partial G}{\partial x_{i}}(t, x, 0) \\
& +\nabla_{\mu} G(t, x, \mathscr{D} u(t, x)) \cdot \mathscr{D} \frac{\partial u(t, x)}{\partial x_{i}}
\end{aligned}
$$

and the above argument that $\left\|\left(\partial \tilde{G} / \partial x_{i}\right)(t)\right\| \leqslant c_{4}|u(t)|_{3}(i=1, \ldots, n)$. Furthermore it follows that $\partial G / \partial x_{i} \in C\left([0, T], L_{2}(\Omega)\right)$, since

$$
\begin{aligned}
& \left\|\left[G\left(t_{1}, ., \mathscr{D} u\left(t_{1}\right)\right)-G\left(t_{1}, ., \mathscr{D} u\left(t_{2}\right)\right)\right] \mathscr{D} \frac{\partial u\left(t_{1}\right)}{\partial x_{i}}\right\| \\
& \leqslant c_{5} \sup _{x \in \Omega}\left|\mathscr{D} u\left(t_{1}, x\right)-\mathscr{D} u\left(t_{2}, x\right)\right|\left\|\mathscr{D} \frac{\partial u\left(t_{1}\right)}{\partial x_{i}}\right\| \\
& \leqslant c_{6}\left\|u\left(t_{1}\right)-u\left(t_{2}\right)\right\|_{(k-1) m}\left\|\mathscr{D} \frac{\partial u\left(t_{1}\right)}{\partial x_{i}}\right\|
\end{aligned}
$$

for $t_{1}, t_{2} \in[0, T]$.

Let (A.10) be proved for $l=0, \ldots, L-1$ with $2 \leqslant L \leqslant(k-3) m$ and consider (A.13). We obtain from the induction hypothesis that

$$
\left.\begin{array}{l}
\frac{\partial G}{\partial x_{i}}(., ., \mathscr{D} u)-\frac{\partial G}{\partial x_{i}}(., ., 0) \in C\left([0, T], H^{L-1}(\Omega)\right), \\
\nabla_{\mu} G(., ., \mathscr{D} u)-\nabla_{\mu} G(., ., 0) \in C\left([0, T], H^{L-1}(\Omega)\right) .
\end{array}\right\}
$$


Obviously it holds that

$$
\left.\begin{array}{l}
\nabla_{\mu} G(., ., 0) \cdot \mathscr{D} \frac{\partial u}{\partial x_{i}} \in \mathscr{C}_{T}^{k-4}, \\
\left|\nabla_{\mu} G(t, ., 0) \cdot \mathscr{D} \frac{\partial u(t)}{\partial x_{i}}\right|_{k-4} \leqslant c|u(t)|_{k-1} \quad \text { for } t \in[0, T] .
\end{array}\right\}
$$

With Lemma A.2 it follows from (A.13)-(A.15) that $\partial G / \partial x_{i} \in C\left([0, T], H^{L-1}(\Omega)\right)$ for $i=1, \ldots, n$. This proves the first part of (A.10) for $l=L$. By an analogous argument we obtain the estimate in (A.10) for $l=L$. Hence (A.10) is proved for $l=0, \ldots,(k-3) m$.

In the next step we prove

$$
\left.\begin{array}{l}
\tilde{G} \in \mathscr{C}_{T}^{l}, \\
|\tilde{G}(t)|_{l}<c\left(1+|u(t)|_{k-1}\right)^{l m}|u(t)|_{k-1} \quad \text { for } t \in[0, T]
\end{array}\right\}
$$

for $l=0, \ldots, k-3$ by induction. In the case $l=0,(\mathrm{~A} .16)$ is already proved by (A.10). For $l=1$, (A.16) follows from (A.12) by the same argument that proves (A.10) in the case $l=1$. Let (A.16) be proved for $l=0, \ldots, L-1$ with $2 \leqslant L \leqslant k-3$ and consider (A.12). The induction hypothesis yields

$$
\begin{gathered}
\left(\partial_{t} G\right)(., ., \mathscr{D} u)-\left(\partial_{t} G\right)(., ., 0) \in \mathscr{C}_{T}^{L-1}, \\
\nabla_{\mu} G(., ., \mathscr{D} u)-\nabla_{\mu} G(., ., 0) \in \mathscr{C}_{T}^{L-1} .
\end{gathered}
$$

Together with

$$
\begin{aligned}
& \nabla_{\mu} G(., ., 0) \cdot \mathscr{D} \frac{\partial u}{\partial t} \in \mathscr{C}_{T}^{k-3}, \\
& \left|\nabla_{\mu} G(., ., 0) \cdot \mathscr{D} \frac{\partial u}{\partial t}\right|_{k-3} \leqslant c|u(t)|_{k}^{\tilde{k}} \quad \text { for } t \in[0, T], \\
& \left|\nabla_{\mu} G(., ., 0) \cdot \mathscr{D} \frac{\partial u}{\partial t}\right|_{k-4} \leqslant c|u(t)|_{k-1} \quad \text { for } t \in[0, T],
\end{aligned}
$$

and Corollary A.3 it follows that

$$
\partial \tilde{G} / \partial t \in \mathscr{C}_{T}^{L-1} \text {. }
$$

Using (A.10) we conclude that $\tilde{G} \in \mathscr{C}_{T}^{L}$. The estimate in (A.16) with $l=L$ can be proved in the same way. Hence (A.16) holds for $l=0, \ldots, k-3$, which proves (A.3). In order to prove $\tilde{G} \in \mathscr{C}_{T}^{k-2}$ and (A.2), we start from (A.16) with $l=k-3$. The argument leading to (A.18) yields $\partial \tilde{G} / \partial t \in \mathscr{C}_{T}^{k-3}$. This implies together with $\tilde{G} \in \mathscr{C}_{T}^{k-3}$ that $\tilde{G} \in \tilde{\mathscr{C}}_{T}^{k-2}$. The estimate (A.2) follows analogously.

In order to prove the second part of Lemma A.1 we note that

$$
\left|G\left(t, x, \mathscr{D} u_{1}(t, x)\right)-G\left(t, x, \mathscr{D} u_{2}(t, x)\right)\right| \leqslant c\left|\mathscr{D} u_{1}(t, x)-\mathscr{D} u_{2}(t, x)\right|
$$

$((t, x) \in[0, T] \times \bar{\Omega})$ and therefore

$$
\| G\left(t, ., \mathscr{D} u_{1}(t)\right)-G\left(t, ., \mathscr{D} u_{2}(t) \| \leqslant c\left|u_{1}(t)-u_{2}(t)\right|_{k-1} .\right.
$$

From this estimate the assertion follows by the same argument as above. 
Corollary A.4. Suppose that $k \geqslant[n / 2 m]+4, R>0$ and $u^{j} \in H^{(k-j) m}(\Omega)$ for $j=0,1,2$ with $\left\|u^{0}\right\|_{k m}+\left\|u^{1}\right\|_{(k-1) m}+\left\|u^{2}\right\|_{(k-2) m} \leqslant R$. Let $G$ satisfy $(A .1)$. We set

$$
G^{*}(x):=G\left(0, x, \bar{D}_{x}^{2 m} u^{0}(x), \bar{D}_{x}^{m} u^{1}(x), u^{2}(x)\right)-G(0, x, 0) .
$$

Then $G^{*} \in H^{(k-2) m}(\Omega)$ and

$$
\left\|G^{*}\right\|_{(k-2) m} \leqslant c\left(\left\|u^{0}\right\|_{k m}+\left\|u^{1}\right\|_{(k-1) m}+\left\|u^{2}\right\|_{(k-2) m}\right),
$$

where $c>0$ depends on $k, R, G$ and $\Omega$.

The proof of Corollary A.4 is the same as the proof of (A.10).

Corollary A.5. Let $k \geqslant k_{2}=[n / 2 m]+4$ and $G$ satisfying $(A .1)$ be given. Furthermore suppose that $u \in \tilde{\mathscr{C}}_{T}^{k} \cap \mathscr{C}_{T}^{k_{2}}$ and $v \in \tilde{\mathscr{C}}_{T}^{k-2}$. Then $G(., ., \mathscr{D} u) v \in \tilde{\mathscr{C}}_{T}^{k-2}$ and

$$
\begin{aligned}
|G(t, ., \mathscr{D} u(t)) v(t)|_{k-3} \leqslant c_{1}(1 & \left.+|u(t)|_{k-1}\right)^{(k-3) m}|u(t)|_{k-1}|v(t)|_{k-3}, \\
\left\|\partial_{t}^{k-2}[G(t, ., \mathscr{D} u(t)) v(t)]\right\| \leqslant & c_{2}\left(1+|u(t)|_{k-1}\right)^{(k-2) m}|u(t)|_{k}|v(t)|_{k-3} \\
& +c_{3}\left\|\partial_{t}^{k-2} v(t)\right\|
\end{aligned}
$$

for $t \in[0, T]$, where $c_{1}, c_{2}, c_{3}>0$ depend only on $k, G$ and $\Omega$.

Proof. The assertion (A.20) can be shown by Lemma A.1, Corollary A.3 and

$$
G(t, ., \mathscr{D} u(t)) v(t)=[G(t, ., \mathscr{D} u(t))-G(t, ., 0)] v(t)+G(t, ., 0) v(t)
$$

$(t \in[0, T])$. Equation (A.21) follows analogously from the Leibnitz rule and Lemma A.2.

Lemma A.6. Let $k \geqslant[n / 2]+1$ and $w_{j}=\left(w_{j}^{(1)}, \ldots, w_{j}^{\left(s_{j}\right)}\right) \in C\left([0, T], H^{k-j}(\Omega)\right)$ for $1 \leqslant j \leqslant k$ be given. If $\gamma_{j} \in \mathbb{N}_{0}^{s_{j}}$ such that

$$
1 \leqslant \sum_{j=1}^{k} j\left|\gamma_{j}\right| \leqslant l
$$

for some $l \leqslant k$, then

$$
\begin{aligned}
& \prod_{j=1}^{l} w_{j}^{y_{j}} \in C\left([0, T], H^{k-l}(\Omega)\right), \\
& \left\|\prod_{j=1}^{l} w_{j}^{y_{j}}(t)\right\|_{k-l} \leqslant \prod_{j=1}^{l}\left\|w_{j}(t)\right\|_{k-j}^{\left|y_{j}\right|} \text { for } t \in[0, T],
\end{aligned}
$$

where

$$
w_{j}^{\gamma_{j}}:=\prod_{i=1}^{s_{j}}\left(w_{j}^{(i)}\right)^{\gamma_{j}^{(i)},} \quad\left\|w_{j}(t)\right\|_{k-j}:=\sum_{i=0}^{s_{j}}\left\|w_{j}^{(i)}(t)\right\|_{k-j}
$$

and the constant $c>0$ depends only on $k, l$ and $\Omega$.

Proof. We perform induction with respect to $l \in \mathbb{N}$. If $l=1$, we have $\gamma_{1}=(0, \ldots, 0,1$, $0, \ldots, 0)$ and (A.23), (A.24) are obvious.

Let (A.23) and (A.24) be proved for $1 \leqslant l \leqslant L-1$ with $L \leqslant k$. We assume that $\gamma_{j} \in \mathbb{N}_{0}^{s_{j}}(j=1, \ldots, L)$ are given such that (A.22) holds with $l=L$. We have the following two cases:

(1) $\left|\gamma_{L}\right|=1$. Then $\left|\gamma_{1}\right|=\cdots=\left|\gamma_{L-1}\right|=0$ and (A.23), (A.24) are obvious. 
(2) $\left|\gamma_{L}\right|=0$. We assume that $\sum_{j=1}^{L-1} j\left|\gamma_{j}\right|=L$. Otherwise the assertion follows directly from the induction hypothesis. Let $J:=\max \left\{j \in \mathbb{N}:\left|\gamma_{j}\right| \neq 0\right\}$. We set

$$
v:=\left(\sum_{j=1}^{J-1} w_{j}^{\gamma^{J}}\right) w_{J}^{\tilde{y}_{j}}
$$

where $\tilde{\gamma}_{J} \in \mathbb{N}_{0}^{s_{J}}$ is chosen so that $\left|\gamma_{J}-\tilde{\gamma}_{J}\right|=1, \tilde{\gamma}_{J} \leqslant \gamma_{J}$. Note that

$$
\sum_{j=1}^{J=1} j\left|\gamma_{j}\right|+J\left|\tilde{\gamma}_{j}\right|=L-J
$$

This implies that $\left|\tilde{\gamma}_{J}\right|=0$ if $J>L-J$ and $\left|\gamma_{j}\right|=0$ if $L-J<j<J-1$. Hence it follows from $w=v w_{j}^{\gamma_{j}}-\tilde{y}_{j}$ together with the induction hypothesis and Lemma A.2 that (A.23) and (A.24) hold for $l=L$. This concludes the proof of Lemma A.6.

Lemma A.7. Let $k \geqslant[n / 2 m]+5$ and suppose that Assumption 2.1 holds and that $u \in \tilde{\mathscr{C}}_{T}^{k}$ is a solution of (1.1)-(1.3), where $f \in \mathscr{C}_{T}^{k-2}$. Then $u \in \mathscr{C}_{T}^{k}$.

Proof. Let $\psi \in C^{\infty}(\Omega)$ with supp $\psi \subset \Omega$. We multiply (1.1) with $\psi$ and differentiate the result with respect to $x_{i}(i=1, \ldots, n)$. This yields

$$
\mathscr{A}_{u}(t)\left(\psi(x) \frac{\partial u(t, x)}{\partial x_{i}}\right)=h(t, x) \quad \text { for }(t, x) \in[0, T] \times \Omega,
$$

where

$$
\begin{aligned}
h(t, x):= & \sum_{|\alpha| \leqslant 2 m} a_{\alpha}(t, x, \mathscr{D} u(t, x))\left[\partial_{x}^{\alpha}\left(\psi(x) \frac{\partial u(t, x)}{\partial x_{i}}\right)-\psi(x) \partial_{x}^{\alpha} \frac{\partial u(t, x)}{\partial x_{i}}\right] \\
& -\psi(x) \mathscr{B}_{u}(t)\left(\frac{\partial}{\partial x_{i}} \partial_{t} u(t, x)\right)-\psi(x) \frac{\partial}{\partial x_{i}} \partial_{t}^{2} u(t, x) \\
& +\left(\frac{\partial F(t, x, \mathscr{D} u(t, x))}{\partial \mu_{2}}\right)^{-1}\left(\frac{\partial}{\partial x_{i}}(\psi(x) f(t, x))\right. \\
& \left.-\psi(x) \frac{\partial F}{\partial x_{i}}(t, x, \mathscr{D} u(t, x))-\frac{\partial \psi(x)}{\partial x_{i}} F(t, x, \mathscr{D} u(t, x))\right)
\end{aligned}
$$

Note that $h \in C\left([0, T], H^{(k-3) m}(\Omega)\right)$. From elliptic regularity theory (compare [6]) we obtain $\psi \partial u / \partial x_{i} \in C\left([0, T], H^{(k-1) m}(\Omega)\right)$. Similar considerations can be made at the boundary of $\Omega$, so that it follows that $u \in C\left([0, T], H^{(k-1) m+1}(\Omega)\right.$ ) (if $\Omega$ has the form (2.1), compare the considerations in [6]). From this and (A.27) we obtain $h \in C([0, T]$, $\left.H^{(k-3) m+1}(\Omega)\right)$. Repeating this step $m-1$ times we conclude that $u \in C([0, T]$, $H^{k m}(\Omega)$ ). Since $u \in \tilde{\mathscr{C}}_{T}^{k}$, this implies $u \in \mathscr{C}_{T}^{k}$.

Lemma A.8. Let $k \geqslant 2$ and suppose that $u^{j} \in H^{(\mathbf{k}-j) m}(\Omega)$ for $j=0, \ldots, k-1$. Then there exists a function $u \in \tilde{\mathscr{C}}_{T}^{k}$ with $\partial_{T}^{j} u(0)=u^{j}$ for $j=0, \ldots, k-1$.

Proof. At first we assume that $k$ is even. Let $\tilde{u}^{j} \in H^{(k-j) m}\left(\mathbb{R}^{n}\right)$ for $j=0, \ldots, k-1$ be extensions of $u^{j}$ onto $\mathbb{R}^{n}$ (compare [1]). We define an operator $A$ by

$$
\left.\begin{array}{rl}
D(A) & :=\left\{v \in L_{2}\left(\mathbb{R}^{n}\right):(-\Delta)^{m} v \in L_{2}\left(\mathbb{R}^{n}\right)\right\}, \\
A v & :=(-\Delta)^{m} v \text { for } v \in D(A) .
\end{array}\right\}
$$


Then $A$ is a positive and self-adjoint in $L_{2}\left(\mathbb{R}^{n}\right)$. Let $v$ be the solution of

$$
\left.\begin{array}{rl}
\left(\prod_{j=1}^{k / 2}\left(\partial_{t}^{2}+j A\right)\right) v=0 & \text { for } t \in[0, T] \\
\partial_{t}^{j} v(0)=\tilde{u}^{j} & \text { for } j=0, \ldots, k-1 .
\end{array}\right\}
$$

It follows by spectral theory and by elliptic regularity theory that (A.29) has a solution

$$
v \in \bigcap_{j=0}^{k} C^{j}\left([0, T], H^{(k-j) m}\left(\mathbb{R}^{n}\right)\right)
$$

The restriction $u$ of $v$ on $\Omega$ is the desired function. Note that $u \in \mathscr{C}_{T}^{k}$.

If $k$ is odd, then we choose $w \in \mathscr{C}_{T}^{k-1}$ such that $\partial_{i}^{j} w(0)=u^{j+1}$ for $j=0, \ldots, k-2$. Then

$$
u(t):=u^{0}+\int_{0}^{t} w(\tau) \mathrm{d} \tau
$$

is the desired function.

\section{Acknowledgements}

The author is grateful to Professor Leis for giving him the opportunity of staying for one and a half years in the stimulating atmosphere of his group.

This paper was partially supported by the Deutsche Forschungsgemeinschaft (SFB 256).

\section{References}

1. Adams, R. A., Sobolev Spaces, Academic Press, New York, 1975.

2. Chen, C. and von Wahl, W., 'Das Rand-Anfangswertproblem für quasilineare Wellengleichungen in Sobolevräumen niedriger Ordnung', J. Reine Angew. Math., 337, 77-112 (1982).

3. Ikawa, M., 'Mixed problems for hyperbolic equations of second order', J. Math. Soc. Japan, 20, 580-608 (1968).

4. Klainerman, S., 'Global existence for nonlinear wave equations', Comm. Pure and Appl. Math., 33, 43-101 (1980).

5. Koch, H., 'Mixed problems for fully nonlinear hyperbolic equations', to appear in Math. $Z$.

6. Lesky, P., Jr, 'Wave equations with time-dependent spatial operators of higher order', to appear in Math. Meth. in the Appl. Sci.

7. Shibatah, Y. and Tsutsumi, Y., 'Local existence of solution for the initial boundary value problem of fully nonlinear wave equation', Nonlin. Anal. Theory, Methods and Appl., 11, 335-365 (1987). 